\title{
Spin Precession of Slow Neutrons in Einstein-Cartan Gravity with Torsion, Chameleon and Magnetic Field
}

\author{
A. N. Ivanov $1, \circledast$ and M. Wellenzohn ${ }^{1,2, \dagger}$ \\ ${ }^{1}$ Atominstitut, Technische Universität Wien, Stadionallee 2, A-1020 Wien, Austria \\ ${ }^{2}$ FH Campus Wien, University of Applied Sciences, Favoritenstraße 226, 1100 Wien, Austria \\ (Dated: October 10, 2018)
}

\begin{abstract}
We analyse a spin precession of slow neutrons in the Einstein-Cartan gravity with torsion, chameleon and magnetic field. For the derivation of the Heisenberg equation of motion of the neutron spin we use the effective low-energy potential, derived by Ivanov and Wellenzohn (Phys. Rev. D 92, 125004 (2015)) for slow neutrons, coupled to gravitational, chameleon and torsion fields to order $1 / m$, where $m$ is the neutron mass. In addition to this low-energy interactions we switch on the interaction of slow neutrons with a magnetic field. We show that to linear order approximation with respect to gravitational, chameleon and torsion fields the Dirac Hamilton operator for fermions (neutrons), moving in spacetimes created by rotating coordinate systems, contains the anti-Hermitian operators of torsion-fermion (neutron) interactions, caused by torsion scalar and tensor space-space-time and time-space-space degrees of freedom. Such anti-Hermitian operators violate $C P$ and $T$ invariance. In the low-energy approximation the $C P$ and $T$ violating torsionfermion (neutron) interactions appear only to order $O(1 / m)$. One may assume that in the rotating Universe and galaxies the obtained anti-Hermitian torsion-fermion interactions might be an origin of i) violation of $C P$ and $T$ invariance in the Universe and ii) of baryon asymmetry. We show that anti-Hermitian torsion-fermion interactions of relativistic fermions, violating $C P$ and $T$ invariance, i) cannot be removed by non-unitary transformations of the Dirac fermion wave functions and ii) are conformal invariant. According to general requirements of conformal invariance of massive particle theories in gravitational fields (see R. H. Dicke, Phys. Rev. 125, 2163 (1962) and A. J. Silenko, Phys. Rev. D 91, 065012 (2015)), conformal invariance of anti-Hermitian torsion-fermion interactions is valid only if the fermion mass is changed by a conformal factor.
\end{abstract}

PACS numbers: 03.65.Pm, 04.25.-g, 04.25.Nx, 14.80.Va

\section{INTRODUCTION}

Recently [1] we have derived to order $O(1 / m)$ the most general effective low-energy potential for slow Dirac fermions with mass $m$, coupled to gravitational, chameleon and torsion fields in the Einstein-Cartan gravity. We have reduced the obtained potential to order $O(1)$ in the large fermion mass expansion. We have shown that the torsion pseudoscalar and tensor degrees of freedom can be, in principle, measured in terrestrial laboratories through minimal torsion-fermion couplings by using rotating devices. This is similar to the experiments by Atwood et al. [2] and by Mashhoon [3]. These experiments used a rotating two-crystal neutron interferometer and a neutron interferometer in a rotating reference frame, respectively. We have assumed that the measurements of the transition frequencies between quantum gravitational states of ultracold neutrons in the qBounce experiments [4]- [9] as functions of an angular velocity $\vec{\omega}$ of a rotating mirror should provide a new level of highly precise probes of the properties of the Einstein-Cartan gravity, dark energy and evolution of the Universe. In turn, the measurements of the phase-shift of slow neutron wave function as a function of an angular velocity $\vec{\omega}$ by a rotating neutron interferometer 10] should be of use for terrestrial probes of new gravitational, chameleon and torsion interactions, derived in [1].

In this paper we propose an analysis of a spin precession of slow neutrons in the Einstein-Cartan gravity with torsion, chameleon and magnetic fields. As has been mentioned by Lehnert, Snow, and Yan [11], a spin precession of slow neutrons is a very sensitive technique to search for possible exotic neutron interactions. In the experiment [11] for the measurement of an upper bound of the linear superposition of constant torsion scalar and pseudoscalar degrees of freedom $\zeta$, caused by torsion-fermion interactions by Kostelecky, Russell, and Tasson [12], a neutron spin rotation in the liquid ${ }^{4} \mathrm{He}$ was investigated. The upper bound $|\zeta|<9.1 \times 10^{-23} \mathrm{GeV}$, obtained by Lehnert, Snow, and Yan [11], is by a factor $10^{5}$ larger compared with the estimate $|\zeta|<10^{-27} \mathrm{GeV}$, obtained in [13] by using the

\footnotetext{
*Electronic address: ivanov@kph.tuwien.ac.at

† Electronic address: max.wellenzohn@gmail.com
} 
estimates in Table I by Kostelecky et al. 12. A spin dynamics of Dirac fermions with mass $m$ in curved spacetimes has been also investigated by Obukhov, Silenko, and Teryaev in [14 16].

The paper is organized as follows. In section [1] we calculate the effective low-energy potential, derived in [1], to linear order in gravitational, chameleon and torsion fields in the spacetime with the Schwarzschild metric, taken in the weak gravitational field approximation and modified by the chameleon field and rotation with an angular velocity $\vec{\omega}$. We show that the linearised effective low-energy potential contains the anti-Hermitian interactions, vanishing at zero angular velocity. In section III we give a detailed analysis of the anti-Hermitian interactions. In section IV we derive the Heisenberg equation of motion for a neutron spin precession in terms of the angular velocity operators, caused by i) a magnetic field, ii) gravitational and chameleon fields and iii) a torsion field, defining Hermitian and anti-Hermitian torsion-fermion interactions. In section $\nabla$ we show that the anti-Hermitian Hamilton operator and, correspondingly, the anti-Hermitian effective low-energy potential of torsion-fermion interactions, violating $C P$ and $T$ invariance, i) cannot be removed by non-unitary (non-Hermitian) transformations [16]-24] (see also [1]), ii) are conformal invariant 20, 21] and, referring to the experiments by Atwood et al. 2] and by Mashhoon 3], iii) can be in principle observable. It should be emphasized that, according to the general requirements of conformal invariance of massive particle theories in gravitational fields under conformal transformation $\tilde{g}_{\mu \nu} \rightarrow O^{2} \check{g}_{\mu \nu}$ (see, for example, Brans and Dicke 25], Dicke 26] and Silenko [21]), anti-Hermitian torsion-fermion interactions are conformal invariant only if the fermion mass $m$ is changed by a conformal factor $O$, i.e $m \rightarrow O^{-1} \check{m}$. In section VI we summarize the obtained results and discuss some possible consequences of the anti-Hermitian torsion-fermion interactions. In the Appendix we give a detailed calculation of the operator $G_{\hat{j}}$, responsible for the anti-Hermitian part of Dirac Hamilton operator and of the effective low-energy potential of slow fermions, coupled to gravitational, chameleon and torsion fields to linear order approximation in curved spacetimes with rotation.

\section{SCHRÖDINGER-PAULI EQUATION FOR SLOW NEUTRONS IN EINSTEIN-CARTAN GRAVITY WITH TORSION, CHAMELEON AND MAGNETIC FIELDS}

The Schrödinger-Pauli equation for slow neutrons, coupled to gravitational, chameleon, torsion and magnetic fields in the Einstein-Cartan gravity [1], is equal to

$$
i \frac{\partial \Psi(t, \vec{r})}{\partial t}=\mathrm{H}_{\mathrm{FW}} \Psi(t, \vec{r})
$$

where $\Psi(t, \vec{r})$ is the wave function of slow neutrons and $\mathrm{H}$ is the Foldy-Wouthuysen Hamilton operator given by

$$
\mathrm{H}_{\mathrm{FW}}=-\frac{1}{2 m} \Delta+m U_{\mathrm{E}}-\vec{\mu} \cdot \vec{B}+\tilde{\Phi}_{\mathrm{eff}}(t, \vec{r}, \vec{S}),
$$

where $m$ is the neutron mass, $\Delta$ is the Laplace operator, $U_{\mathrm{E}}=\vec{g} \cdot \vec{r}$ is the gravitational potential of the Earth with the Newtonian gravitational acceleration $\vec{g}$, and $\vec{B}$ is a magnetic field. The neutron magnetic dipole moment $\vec{\mu}=2 \kappa_{n} \mu_{N} \vec{S}$ [27] is expressed in terms of the neutron anomalous magnetic moment $\kappa_{n}=-1.9130427(5)$, measured in nuclear magnetons $\mu_{N}=e / 2 m_{p}=3.1524512605(22) \times 10^{-8} \mathrm{eV} \mathrm{T}^{-1}$ [28], which is defined in terms of the electric charge $e$ and mass $m_{p}$ of the proton, and the neutron spin operator $\vec{S}=\frac{1}{2} \vec{\sigma}$, where $\vec{\sigma}$ are the $2 \times 2$ Pauli matrices [29]. Then, $\tilde{\Phi}_{\text {eff }}(t, \vec{r}, \vec{S})=\Phi_{\text {eff }}(t, \vec{r}, \vec{S})-U_{\mathrm{E}}$, where $\Phi_{\text {eff }}(t, \vec{r}, \vec{S})$ is the effective low-energy potential for slow neutrons, coupled to gravitational, chameleon and torsion fields (see Eq.(A.15) of Ref.[1]). We would like to note that we have included the interaction of slow neutrons with a magnetic field to linear order approximation of the magnetic field. Since below we analyse the contributions of the effective low-energy potential $\Phi_{\text {eff }}(t, \vec{r}, \vec{S})$ to linear order approximation of gravitational, chameleon and torsion fields, we have neglected all interactions, containing the products of a magnetic field with gravitational, chameleon and torsion fields.

The calculation of the effective low-energy potential $\Phi_{\mathrm{eff}}(t, \vec{r}, \vec{S})$ we perform in the curved spacetime with the line element [1] (see also [15])

$$
d \tilde{s}^{2}=\tilde{V}^{2}(x) d t^{2}+\eta_{\hat{j} \ell} \tilde{W}_{j}^{\hat{j}}(x) \tilde{W}_{\ell}^{\hat{\ell}}(x)\left(d x^{j}-K^{j}(x) d t\right)\left(d x^{\ell}-K^{\ell}(x) d t\right),
$$

where $\hat{j}=1,2,3$ and $j=1,2,3$ are indices of the Minkowski and curved spacetime, respectively, $\eta_{\hat{j} \hat{\ell}}$ is a spatial part of the metric tensor in the Minkowski spacetime. The functions $\tilde{V}^{2}(x)$ and $\tilde{W}_{j}^{\hat{j}}(x)$ are defined by an arbitrary gravitational field. In comparison with Obukhov, Silenko, and Teryaev [15] the functions $\tilde{V}^{2}(x)$ and $\tilde{W}_{j}^{\hat{j}}(x)$ are modified by the chameleon field. In turn, the functions $K^{j}(x)$, caused by rotations, are not modified by the chameleon 
field. The vierbein fields in terms of which slow neutrons couple to gravitational, chameleon and torsion fields in the Einstein-Cartan gravity with the metric tensor in Eq.(3) are equal to [1]

$$
\begin{aligned}
& \tilde{e}_{0}^{\hat{0}}(x)=\tilde{V}(x), \tilde{e}_{0}^{\hat{j}}(x)=-\tilde{W}_{j}^{\hat{j}}(x) K^{j}(x), \tilde{e}_{j}^{\hat{0}}(x)=0, \tilde{e}_{j}^{\hat{j}}(x)=\tilde{W}_{j}^{\hat{j}}(x), \\
& \tilde{e}_{\hat{0}}^{0}(x)=\frac{1}{\tilde{V}(x)}, \tilde{e}_{\hat{j}}^{0}(x)=0, \tilde{e}_{\hat{0}}^{j}(x)=\frac{K^{j}(x)}{\tilde{V}(x)}, \tilde{e}_{\hat{j}}^{j}(x)=\tilde{W}_{\hat{j}}^{j}(x) .
\end{aligned}
$$

The vierbein fields in Eq.(44) have been calculated at the assumption that the functions $\tilde{W}_{j}^{\hat{j}}(x)$ and $\tilde{W}_{\hat{j}}^{j}(x)$ obey the orthogonality relations [1]

$$
\tilde{W}_{j}^{\hat{j}}(x) \tilde{W}_{\hat{\ell}}^{j}=\delta_{\hat{\ell}}^{\hat{j}}, \tilde{W}_{\hat{j}}^{j}(x) \tilde{W}_{\ell}^{\hat{j}}=\delta_{\ell}^{j},
$$

which are fulfilled for the Schwarzschild metric in the weak gravitational field approximation [13]. In terms of the vierbein fields Eq. (4) the effective low-energy potential $\Phi_{\text {eff }}(t, \vec{r}, \vec{S})$ is given by

$$
\begin{aligned}
& \Phi_{\text {eff }}(t, \vec{r}, \vec{S})=(A-1) m+B+2 C^{\hat{\ell}} S_{\hat{\ell}}+i L^{j} \frac{\partial}{\partial x^{j}}+\frac{1}{2 m} \eta^{\hat{j} \hat{k}}\left(\frac{D_{\hat{j}}^{j} D_{\hat{k}}^{k}}{A}-\delta_{\hat{j}}^{j} \delta_{\hat{k}}^{k}\right) \frac{\partial^{2}}{\partial x^{j} \partial x^{k}} \\
& +\frac{1}{4 m A} \eta^{\hat{j} \hat{k}} D_{\hat{j}}^{j} \frac{\partial D_{\hat{k}}^{k}}{\partial x^{j}} \frac{\partial}{\partial x^{k}}+\frac{1}{4 m} \eta^{\hat{j} \hat{k}} D_{\hat{k}}^{k} \frac{\partial}{\partial x^{k}}\left(\frac{D_{\hat{j}}^{j}}{A}\right) \frac{\partial}{\partial x^{j}}+\frac{1}{2 m A} i \epsilon^{\hat{j} \hat{k} \hat{\ell}} S_{\hat{\ell}} D_{\hat{j}}^{j} \frac{\partial D_{\hat{k}}^{k}}{\partial x^{j}} \frac{\partial}{\partial x^{k}} \\
& -\frac{1}{2 m} i \epsilon^{\hat{j} \hat{k} \hat{\ell}} S_{\hat{\ell}} D_{\hat{k}}^{k} \frac{\partial}{\partial x^{k}}\left(\frac{D_{\hat{j}}^{j}}{A}\right) \frac{\partial}{\partial x^{j}}+\frac{1}{2 m A} \eta^{\hat{j} \hat{k}} G_{\hat{j}} D_{\hat{k}}^{k} \frac{\partial}{\partial x^{k}}+\frac{1}{4 m} \eta^{\hat{j} \hat{k}} D_{\hat{k}}^{k} \frac{\partial}{\partial x^{k}}\left(\frac{G_{\hat{j}}}{A}\right) \\
& -\frac{1}{2 m} i \epsilon^{\hat{j} \hat{k} \hat{\ell}} S_{\hat{\ell}} D_{\hat{k}}^{k} \frac{\partial}{\partial x^{k}}\left(\frac{G_{\hat{j}}}{A}\right)+\frac{1}{m A} i S^{\hat{k}} K D_{\hat{k}}^{k} \frac{\partial}{\partial x^{k}}+\frac{1}{2 m} i S^{\hat{k}} D_{\hat{k}}^{k} \frac{\partial}{\partial x^{k}}\left(\frac{K}{A}\right)+\frac{1}{2 m A} \eta^{\hat{j} \hat{k}} G_{\hat{k}} D_{\hat{j}}^{j} \frac{\partial}{\partial x^{j}} \\
& +\frac{1}{4 m A} \eta^{\hat{j} \hat{k}} D_{\hat{j}}^{j} \frac{\partial G_{\hat{k}}}{\partial x^{j}}+\frac{1}{2 m A} i \epsilon^{\hat{j} \hat{k} \hat{\ell}} S_{\hat{\ell}} D_{\hat{j}}^{j} \frac{\partial G_{\hat{k}}}{\partial x^{j}}+\frac{1}{2 m A} \eta^{\hat{j} \hat{k}} G_{\hat{j}} G_{\hat{k}}+\frac{1}{m A} i S^{\hat{k}} K G_{\hat{k}}+\frac{1}{m A} i S^{\hat{j}} K D_{\hat{j}}^{j} \frac{\partial}{\partial x^{j}} \\
& +\frac{1}{2 m A} i S^{\hat{j}} D_{\hat{j}}^{j} \frac{\partial K}{\partial x^{j}}+\frac{1}{m A} i S^{\hat{j}} K G_{\hat{j}}+\frac{1}{2 m A} K^{2}-\frac{1}{4 m A^{2}} \eta^{\hat{j} \hat{k}} D_{\hat{j}}^{j} D_{\hat{k}}^{k} \frac{\partial A}{\partial x^{k}} \frac{\partial}{\partial x^{j}}-\frac{1}{8 m A} \eta^{j \hat{j} \hat{k}} D_{\hat{j}}^{j} \frac{\partial}{\partial x^{j}}\left(\frac{D_{\hat{k}}^{k}}{A} \frac{\partial A}{\partial x^{k}}\right) \\
& -\frac{1}{4 m A} i \epsilon^{\hat{j} \hat{k} \hat{\ell}} S_{\hat{\ell}} D_{\hat{j}}^{j} \frac{\partial}{\partial x^{j}}\left(\frac{D_{\hat{k}}^{k}}{A} \frac{\partial A}{\partial x^{k}}\right)-\frac{1}{4 m A^{2}} \eta^{\hat{j} \hat{k}} G_{\hat{j}} D_{\hat{k}}^{k} \frac{\partial A}{\partial x^{k}}-\frac{1}{2 m A^{2}} i S^{\hat{k}} K D_{\hat{k}}^{k} \frac{\partial A}{\partial x^{k}}+\frac{1}{4 m A^{3}} \eta^{\hat{j} \hat{k}} D_{\hat{j}}^{j} D_{\hat{k}}^{k} \frac{\partial A}{\partial x^{j}} \frac{\partial A}{\partial x^{k}} .
\end{aligned}
$$

The operators $A, B, C^{\hat{\ell}}, D_{\hat{j}}^{j}, G_{\hat{j}}, K$ and $L^{j}$ are equal to [1]

$$
\begin{aligned}
A & =\tilde{e}_{0}^{\hat{0}}(x), \\
B & =-\frac{1}{2} i \tilde{e}_{0}^{\hat{0}}(x) \frac{1}{\sqrt{-\tilde{g}}} \frac{\partial}{\partial x^{j}}\left(\sqrt{-\tilde{g}} \tilde{e}_{\hat{0}}^{j}(x)\right)+\frac{1}{2} i\left(\tilde{e}_{0}^{\hat{0}}(x)\right)^{2} \tilde{e}_{\hat{0}}^{j}(x) \frac{1}{\sqrt{-\tilde{g}(x)}} \frac{\partial}{\partial x^{j}}\left(\sqrt{-\tilde{g}(x)} \tilde{e}_{\hat{0}}^{0}(x)\right), \\
C^{\hat{\ell}} & =\frac{1}{4} \tilde{e}_{0}^{\hat{0}}(x)\left(\tilde{\omega}_{0 \hat{j} \hat{k}}(x) \tilde{e}_{\hat{0}}^{0}(x)+\tilde{\omega}_{\ell \hat{j} \hat{k}}(x) \tilde{e}_{\hat{0}}^{\ell}(x)+\tilde{\omega}_{\ell \hat{0} \hat{j}}(x) \tilde{e}_{\hat{k}}^{\ell}(x)-\tilde{\omega}_{\ell \hat{j} \hat{0}}(x) \tilde{e}_{\hat{k}}^{\ell}(x)\right) \epsilon^{\hat{j} \hat{k} \hat{\ell}}, \\
D_{\hat{j}}^{j} & =-\tilde{e}_{0}^{\hat{0}}(x) \tilde{e}_{\hat{j}}^{j}(x), \\
G_{\hat{j}} & =\frac{1}{2} \tilde{e}_{0}^{\hat{0}}(x)\left(\tilde{\mathcal{T}}_{\alpha \ell}^{\alpha}(x) \tilde{e}_{\hat{j}}^{\ell}(x)+\tilde{\omega}_{0 \hat{j} \hat{0}}(x) \tilde{e}_{\hat{0}}^{0}(x)+\tilde{\omega}_{\ell \hat{j} \hat{0}}(x) \tilde{e}_{\hat{0}}^{\ell}(x)+\tilde{\omega}_{\ell \hat{j} \hat{k}}(x) \tilde{e}_{\hat{\ell}}^{\ell}(x) \eta^{\hat{\ell} \hat{k}}\right) \\
& +\frac{1}{2}\left(\tilde{e}_{0}^{\hat{0}}(x)\right)^{2} \tilde{e}_{\hat{j}}^{j}(x) \frac{1}{\sqrt{-\tilde{g}(x)}} \frac{\partial}{\partial x^{j}}\left(\sqrt{-\tilde{g}(x)} \tilde{e}_{\hat{0}}^{0}(x)\right), \\
K & =-\frac{1}{4} \tilde{\omega}_{\ell \hat{j} \hat{k}}(x) \tilde{e}_{0}^{\hat{0}}(x) \tilde{e}_{\hat{\ell}}^{\ell}(x) \epsilon^{\hat{j} \hat{k} \hat{\ell}}, \\
L^{j} & =-\tilde{e}_{0}^{\hat{0}}(x) \tilde{e}_{\hat{0}}^{j}(x) .
\end{aligned}
$$

Here $\tilde{g}(x)=-\operatorname{det}\left\{\tilde{g}_{\mu \nu}(x)\right\}$, where the metric tensor in the Jordan frame $\tilde{g}_{\mu \nu}(x)$ is related to the metric tensor in the Einstein frame $g_{\mu \nu}(x)$ by $\tilde{g}_{\mu \nu}(x)=f^{2}(x) g_{\mu \nu}(x)$ [30, 31], where the conformal factor $f(x)=e^{\beta \phi(x) / M_{\mathrm{Pl}}}$ is defined in terms of the chameleon field $\phi(x)$, the chameleon-matter coupling constant $\beta$ and the reduced Planck mass $M_{\mathrm{Pl}}=1 / \sqrt{8 \pi G_{N}}=2.435 \times 10^{27} \mathrm{eV}$ with the Newtonian gravitational constant $G_{N}[28]$. The spin connection $\tilde{\omega}_{\mu \hat{\alpha} \hat{\beta}}(x)$ is defined by $[1,13$ ]

$$
\tilde{\omega}_{\mu \hat{\alpha} \hat{\beta}}(x)=-\eta_{\hat{\alpha} \hat{\varphi}}\left(\partial_{\mu} \tilde{e}_{\nu}^{\hat{\varphi}}(x)-\tilde{\Gamma}_{\mu \nu}^{\alpha}(x) \tilde{e}_{\alpha}^{\hat{\varphi}}(x)\right) \tilde{e}_{\hat{\beta}}^{\nu}(x)
$$


where $\alpha=0,1,2,3$ and $\hat{\alpha}=0,1,2,3$ are the indices in the 4 -dimensional curved and Minkowski spacetime, respectively. The affine connection $\tilde{\Gamma}_{\mu \nu}^{\alpha}(x)$ is determined by

$$
\tilde{\Gamma}_{\mu \nu}^{\alpha}(x)=\widetilde{\left\{{ }_{\mu \nu}^{\alpha}\right\}}+\tilde{\mathcal{K}}_{\mu \nu}^{\alpha}(x)
$$

where $\widetilde{\left\{\alpha_{\mu \nu}\right\}}$ are the Christoffel symbols 32 ]

$$
\widetilde{\left\{{ }_{\mu \nu}\right\}}=\frac{1}{2} \tilde{g}^{\alpha \lambda}\left(\frac{\partial \tilde{g}_{\lambda \mu}}{\partial x^{\nu}}+\frac{\partial \tilde{g}_{\lambda \nu}}{\partial x^{\mu}}-\frac{\partial \tilde{g}_{\mu \nu}}{\partial x^{\lambda}}\right)
$$

and $\tilde{\mathcal{K}}^{\alpha}{ }_{\mu \nu}(x)=-\frac{1}{2}\left(\tilde{\mathcal{T}}^{\alpha}{ }_{\mu \nu}(x)-\tilde{\mathcal{T}}_{\mu}{ }^{\alpha}{ }_{\nu}(x)-\tilde{\mathcal{T}}_{\nu}{ }^{\alpha}{ }_{\mu}(x)\right)$ is the contorsion tensor, expressed in terms of the torsion field $\tilde{\mathcal{T}}^{\alpha}{ }_{\mu \nu}(x)=\tilde{g}^{\alpha \sigma}(x) \tilde{\mathcal{T}}_{\sigma \mu \nu}(x)$ [13]. For the analysis of the effective potential Eq.(6) we assume a motion of Dirac fermions with mass $m$ in the curved spacetime with the Schwarzschild metric, taken in the weak gravitational field approximation and modified by the contributions of the chameleon field and rotation. The line element of such a spacetime is given by $[1$

$$
d \tilde{s}^{2}=\left(1+2 U_{+}\right) d t^{2}+2\left(1-2 U_{-}\right) \vec{K} \cdot d \vec{r} d t-\left(1-2 U_{-}\right) d \vec{r}^{2},
$$

where we have neglected the contribution of the terms of order $\vec{K}^{2}$ that is well justified in terrestrial laboratories 33 and kept the contributions of the chameleon field to linear order. The potentials $U_{ \pm}$are equal to [13]

$$
U_{ \pm}=U_{\mathrm{E}} \pm \frac{\beta}{M_{\mathrm{Pl}}} \phi(x)
$$

To linear order contributions of the gravitational and chameleon field the vierbein fields Eq.(4) read

$$
\begin{aligned}
& \tilde{e}_{0}^{\hat{0}}(x)=1+U_{+}, \tilde{e}_{0}^{\hat{j}}(x)=-\left(1-U_{-}\right) K^{\hat{j}}(x), \tilde{e}_{j}^{\hat{0}}(x)=0, \tilde{e}_{j}^{\hat{j}}(x)=\left(1-U_{-}\right) \delta_{j}^{\hat{j}}, \\
& \tilde{e}_{\hat{0}}^{0}(x)=1-U_{+}, \tilde{e}_{\hat{0}}^{j}(x)=+\left(1-U_{+}\right) K^{j}(x), \tilde{e}_{\hat{j}}^{0}(x)=0, \tilde{e}_{\hat{j}}^{j}(x)=\left(1+U_{-}\right) \delta_{\hat{j}}^{j} .
\end{aligned}
$$

In the spacetime with metric Eq.(11) and the vierbein fields Eq.(13) the operators $A, B, C^{\hat{\ell}}, D_{\hat{j}}^{j}, G_{\hat{j}}, K$ and $L^{j}$, calculated to linear approximation in gravitational, chameleon and torsion fields, are equal to

$$
\begin{aligned}
A & =1+U_{+}, \\
B & =-\frac{1}{2} i \operatorname{div} \vec{K} \\
C^{\hat{\ell}} & =-\frac{1}{4}(\operatorname{rot} \vec{K})^{\hat{\ell}}+\frac{1}{8} \epsilon^{\hat{\ell} \hat{j} \hat{k}}\left(\mathcal{T}_{\hat{j} \hat{k} \hat{0}}+\mathcal{T}_{\hat{k} \hat{0} \hat{j}}+\mathcal{T}_{\hat{0} \hat{j} \hat{k}}\right)+\frac{1}{4} \epsilon^{\hat{\ell} \hat{j} \hat{k}} K_{\hat{j}} \mathcal{T}_{\hat{0} \hat{0} \hat{k}}+\frac{1}{4} \epsilon^{\hat{\ell} \hat{j} \hat{k}} \mathcal{T}_{\hat{j} \hat{k} \hat{a}} K^{\hat{a}}= \\
& =-\frac{1}{4}(\operatorname{rot} \vec{K})^{\hat{\ell}}+\frac{1}{4} \mathcal{B}^{\hat{\ell}}+\frac{1}{6} \mathcal{K} K^{\hat{\ell}}+\frac{1}{4} \epsilon^{\hat{\ell} \hat{j} \hat{k}} K_{\hat{j}} \mathcal{M}_{\hat{0} \hat{0} \hat{k}}+\frac{1}{4} \epsilon^{\hat{\ell} \hat{j} \hat{k}} \mathcal{M}_{\hat{j} \hat{k} \hat{a}} K^{\hat{a}}, \\
D_{\hat{j}}^{j} & =-\left(1+U_{+}+U_{-}\right) \delta_{\hat{j}}^{j}, \\
G_{\hat{j}} & =-\frac{1}{2} \frac{\partial}{\partial x^{\hat{j}}}\left(U_{+}+U_{-}\right)+\frac{1}{2}\left(\mathcal{T}_{\hat{0} \hat{j} \hat{\ell}}+\mathcal{T}_{\hat{\ell} \hat{j} \hat{0}}\right) K^{\hat{\ell}}+\frac{1}{2} K_{\hat{j}} \mathcal{T}_{\hat{\ell} \hat{k} \hat{0} \hat{l}} \eta^{\hat{\ell} \hat{k}}= \\
& =-\frac{1}{2} \frac{\partial}{\partial x^{\hat{j}}}\left(U_{+}+U_{-}\right)+\frac{2}{3} \mathcal{E}_{\hat{0}} K_{\hat{j}}+\frac{1}{2}\left(\mathcal{M}_{\hat{\ell} \hat{j} \hat{0}}+\mathcal{M}_{\hat{0} \hat{j} \hat{\ell}}\right) K^{\hat{\ell}}, \\
K^{\prime} & =-\frac{1}{8} \epsilon^{\hat{j} \hat{k} \hat{\ell}} \mathcal{T}_{\hat{j} \hat{k} \hat{\ell}}+\frac{1}{8} \epsilon^{\hat{j} \hat{k} \hat{\ell}} K_{\hat{j}} \mathcal{T}_{\hat{0} \hat{k} \hat{\ell}}= \\
& =-\frac{1}{4} \mathcal{K}+\frac{1}{12} \vec{K} \cdot \overrightarrow{\mathcal{B}}+\frac{1}{8} \epsilon^{\hat{j} \hat{k} \hat{\ell}} K_{\hat{j}} \mathcal{M}_{\hat{0} \hat{k} \hat{\ell}}, \\
L^{j} & =-K^{j},
\end{aligned}
$$

where $\mathcal{B}^{\hat{\ell}}=\frac{1}{2} \epsilon^{\hat{\ell} \hat{j} \hat{k}}\left(\mathcal{T}_{\hat{j} \hat{k} \hat{0}}+\mathcal{T}_{\hat{k} \hat{0} \hat{j}}+\mathcal{T}_{\hat{0} \hat{j} \hat{k}}\right), \mathcal{K}=\frac{1}{2} \epsilon^{\hat{j} \hat{k} \hat{\ell}} \mathcal{T}_{\hat{j} \hat{k} \hat{\ell} \hat{\ell}}, \mathcal{M}_{\hat{\sigma} \hat{\mu} \hat{\nu}}\left(\mathcal{M}_{\hat{0} \hat{0} \hat{k}}, \mathcal{M}_{\hat{0} \hat{j} \hat{\ell}}, \mathcal{M}_{\hat{\ell} \hat{j} \hat{0}}\right.$ and $\left.\mathcal{M}_{\hat{j} \hat{k} \hat{\ell}}\right)$ and $\mathcal{E}_{\hat{0}}=\mathcal{T}^{\hat{\alpha}}{ }_{\hat{\alpha} \hat{0}}$ are torsion axial-vector, pseudoscalar, tensor and scalar degrees of freedom, respectively [1, 13]. For the calculation of the operators in Eq.(14) we have used the following irreducible representation of the torsion field $\mathcal{T}_{\sigma \mu \nu}$ [12] (see also $[1,13]$ )

$$
\mathcal{T}_{\hat{\sigma} \hat{\mu} \hat{\nu}}(x)=\frac{1}{3}\left(\eta_{\hat{\sigma} \hat{\mu}} \mathcal{E}_{\hat{\nu}}(x)-\eta_{\hat{\sigma} \hat{\nu}} \mathcal{E}_{\hat{\mu}}(x)\right)+\frac{1}{3} \epsilon_{\hat{\sigma} \hat{\mu} \hat{\nu} \hat{\alpha}} \mathcal{B}^{\hat{\alpha}}(x)+\mathcal{M}_{\hat{\sigma} \hat{\mu} \hat{\nu}}(x) .
$$


The torsion field $\mathcal{T}_{\hat{\sigma} \hat{\mu} \hat{\nu}}(x)$, antisymmetric with respect to indices $\hat{\mu}$ and $\hat{\nu}$, possesses 24 independent components, where the 4 -vector $\mathcal{E}_{\hat{\nu}}(x)$ and axial 4 -vector $\mathcal{B}^{\hat{\alpha}}(x)$ fields with 4 independent degrees of freedom each are defined by

$$
\mathcal{E}_{\hat{\nu}}(x)=\eta^{\hat{\sigma} \hat{\mu}} \mathcal{T}_{\hat{\sigma} \hat{\mu} \hat{\nu}}(x) \quad, \quad \mathcal{B}^{\hat{\alpha}}(x)=\frac{1}{2} \epsilon^{\hat{\alpha} \hat{\sigma} \hat{\mu} \hat{\nu}} \mathcal{T}_{\hat{\sigma} \hat{\mu} \hat{\nu}}(x) .
$$

The residual 16 degrees of freedom are absorbed by the tensor $\mathcal{M}_{\hat{\alpha} \hat{\mu} \hat{\nu}}$, which obeys the constraints $\eta^{\hat{\sigma} \hat{\mu}} \mathcal{M}_{\hat{\sigma} \hat{\mu} \hat{\nu}}=$ $\epsilon^{\hat{\alpha} \hat{\sigma} \hat{\mu} \hat{\nu}} \mathcal{M}_{\hat{\sigma} \hat{\mu} \hat{\nu}}=0$. Then, $\epsilon_{\hat{\sigma} \hat{\mu} \hat{\nu} \hat{\alpha}}$ and $\epsilon^{\hat{\alpha} \hat{\sigma} \hat{\mu} \hat{\nu}}$ are the Levi-Civita tensors such as $\epsilon_{\hat{0} \hat{1} \hat{z} \hat{z}}=-\epsilon^{\hat{0} \hat{1} \hat{2} \hat{3}}=-1[29]$. For the derivation of the axial-vector field $\mathcal{B}^{\hat{\alpha}}$ in terms of the torsion field $\mathcal{T}_{\hat{\sigma} \hat{\mu} \hat{\nu}}(x)$ we have used the relation $\epsilon^{\hat{\alpha} \hat{\sigma} \hat{\mu} \hat{\nu}} \epsilon_{\hat{\sigma} \hat{\mu} \hat{\nu} \hat{\beta}}=-6 \delta_{\hat{\beta}}^{\hat{\alpha}}$ [29]. Now we rewrite the effective low-energy potential Eq. (6) omitting the terms, which contributions are smaller compared to the terms of the linear order approximation

$$
\begin{aligned}
& \Phi_{\mathrm{eff}}(t, \vec{r}, \vec{S})=(A-1) m+B+2 C^{\hat{\ell}} S_{\hat{\ell}}+i L^{j} \frac{\partial}{\partial x^{j}}+\frac{1}{2 m} \eta^{\hat{j} \hat{k}}\left(\frac{D_{\hat{j}}^{j} D_{\hat{k}}^{k}}{A}-\delta_{\hat{j}}^{j} \delta_{\hat{k}}^{k}\right) \frac{\partial^{2}}{\partial x^{j} \partial x^{k}} \\
& +\frac{1}{4 m A} \eta^{\hat{j} \hat{k}} D_{\hat{j}}^{j} \frac{\partial D_{\hat{k}}^{k}}{\partial x^{j}} \frac{\partial}{\partial x^{k}}+\frac{1}{4 m} \eta^{\hat{j} \hat{k}} D_{\hat{k}}^{k} \frac{\partial}{\partial x^{k}}\left(\frac{D_{\hat{j}}^{j}}{A}\right) \frac{\partial}{\partial x^{j}}+\frac{1}{2 m A} i \epsilon^{\hat{j} \hat{k} \hat{\ell}} S_{\hat{\ell}} D_{\hat{j}}^{j} \frac{\partial D_{\hat{k}}^{k}}{\partial x^{j}} \frac{\partial}{\partial x^{k}} \\
& -\frac{1}{2 m} i \epsilon^{\hat{j} \hat{k} \hat{\ell}} S_{\hat{\ell}} D_{\hat{k}}^{k} \frac{\partial}{\partial x^{k}}\left(\frac{D_{\hat{j}}^{j}}{A}\right) \frac{\partial}{\partial x^{j}}+\frac{1}{2 m A} \eta^{\hat{j} \hat{k}} G_{\hat{j}} D_{\hat{k}}^{k} \frac{\partial}{\partial x^{k}}+\frac{1}{4 m} \eta^{\hat{j} \hat{k}} D_{\hat{k}}^{k} \frac{\partial}{\partial x^{k}}\left(\frac{G_{\hat{j}}}{A}\right) \\
& -\frac{1}{2 m} i \epsilon^{\hat{j} \hat{k} \hat{\ell}} S_{\hat{\ell}} D_{\hat{k}}^{k} \frac{\partial}{\partial x^{k}}\left(\frac{G_{\hat{j}}}{A}\right)+\frac{1}{m A} i S^{\hat{k}} K D_{\hat{k}}^{k} \frac{\partial}{\partial x^{k}}+\frac{1}{2 m} i S^{\hat{k}} D_{\hat{k}}^{k} \frac{\partial}{\partial x^{k}}\left(\frac{K}{A}\right)+\frac{1}{2 m A} \eta^{\hat{j} \hat{k}} G_{\hat{k}} D_{\hat{j}}^{j} \frac{\partial}{\partial x^{j}} \\
& +\frac{1}{4 m A} \eta^{\hat{j} \hat{k}} D_{\hat{j}}^{j} \frac{\partial G_{\hat{k}}}{\partial x^{j}}+\frac{1}{2 m A} i \epsilon^{\hat{j} \hat{k} \hat{\ell}} S_{\hat{\ell}} D_{\hat{j}}^{j} \frac{\partial G_{\hat{k}}}{\partial x^{j}}+\frac{1}{m A} i S^{\hat{j}} K D_{\hat{j}}^{j} \frac{\partial}{\partial x^{j}}+\frac{1}{2 m A} i S^{\hat{j}} D_{\hat{j}}^{j} \frac{\partial K}{\partial x^{j}} \\
& -\frac{1}{4 m A^{2}} \eta^{\hat{j} \hat{k}} D_{\hat{j}}^{j} D_{\hat{k}}^{k} \frac{\partial A}{\partial x^{k}} \frac{\partial}{\partial x^{j}}-\frac{1}{8 m A} \eta^{\hat{j} \hat{k}} D_{\hat{j}}^{j} \frac{\partial}{\partial x^{j}}\left(\frac{D_{\hat{k}}^{k}}{A} \frac{\partial A}{\partial x^{k}}\right)-\frac{1}{4 m A} i \epsilon^{\hat{j} \hat{k} \hat{\ell}} S_{\hat{\ell}} D_{\hat{j}}^{j} \frac{\partial}{\partial x^{j}}\left(\frac{D_{\hat{k}}^{k}}{A} \frac{\partial A}{\partial x^{k}}\right) .
\end{aligned}
$$

Plugging the operators Eq.(14) into Eq.(17) we arrive at the following effective low-energy potential

$$
\tilde{\Phi}_{\mathrm{eff}}(t, \vec{r}, \vec{S})=\Phi_{\mathrm{eff}}^{(1)}(t, \vec{r}, \vec{S})+\Phi_{\mathrm{eff}}^{(2)}(t, \vec{r}, \vec{S})+\Phi_{\mathrm{eff}}^{(3)}(t, \vec{r}, \vec{S})+\Phi_{\mathrm{eff}}^{(4)}(t, \vec{r}, \vec{S}),
$$

where we have denoted:

$$
\begin{aligned}
\Phi_{\mathrm{eff}}^{(1)}(t, \vec{r}, \vec{S}) & =m\left(U_{+}-U_{\mathrm{E}}\right)-i \vec{K} \cdot \vec{\nabla}-i \frac{1}{2} \operatorname{div} \vec{K}+\frac{1}{2} \vec{S} \cdot \operatorname{rot} \vec{K}-\frac{1}{2} \vec{S} \cdot \overrightarrow{\mathcal{B}} \\
& -\frac{1}{3} \mathcal{K} \vec{S} \cdot \vec{K}-\frac{1}{2} \vec{S} \cdot(\vec{K} \times \overrightarrow{\mathcal{M}})+\frac{1}{2} S_{\hat{j}} \epsilon^{\hat{j} \hat{k} \hat{\ell}} \mathcal{M}_{\hat{k} \hat{\ell} \hat{a}} K^{\hat{a}}
\end{aligned}
$$

with $(\overrightarrow{\mathcal{M}})_{\hat{k}}=-\mathcal{M}_{\hat{0} \hat{0} \hat{k}}$. The effective low-energy potential Eq.(19) agrees well with the result, obtained in [1]. Then, the potential $\Phi_{\mathrm{eff}}^{(2)}(t, \vec{r}, \vec{S})$, taking the form

$$
\begin{aligned}
\Phi_{\mathrm{eff}}^{(2)}(t, \vec{r}, \vec{S})= & -\frac{1}{2 m} \vec{\nabla}\left(U_{+}+2 U_{-}\right) \cdot \vec{\nabla}-\frac{1}{2 m}\left(U_{+}+2 U_{-}\right) \Delta-\frac{1}{8 m} \Delta\left(U_{+}+2 U_{-}\right)-\frac{i}{2 m} \vec{S} \cdot\left(\vec{\nabla}\left(U_{+}+2 U_{-}\right) \times \vec{\nabla}\right) \\
& +\frac{i}{2 m} \mathcal{K} \vec{S} \cdot \vec{\nabla}+\frac{i}{4 m} \vec{S} \cdot \vec{\nabla} \mathcal{K},
\end{aligned}
$$

reproduces the results, obtained in [13]. The potentials $\Phi_{\mathrm{eff}}^{(3)}(t, \vec{r}, \vec{S})$ and $\Phi_{\mathrm{eff}}^{(4)}(t, \vec{r}, \vec{S})$ are equal to

$$
\Phi_{\mathrm{eff}}^{(3)}(t, \vec{r}, \vec{S})=-\frac{i}{6 m}(\vec{K} \cdot \overrightarrow{\mathcal{B}}) \vec{S} \cdot \vec{\nabla}-\frac{i}{12 m} \vec{S} \cdot \vec{\nabla}(\vec{K} \cdot \overrightarrow{\mathcal{B}})-\frac{i}{4 m}\left(\epsilon^{\hat{j} \hat{k} \hat{\ell}} K_{\hat{j}} \mathcal{M}_{\hat{0} \hat{k} \hat{\ell}}\right) \vec{S} \cdot \vec{\nabla}-\frac{i}{8 m} \vec{S} \cdot \vec{\nabla}\left(\epsilon^{\hat{j} \hat{k} \hat{\ell}} K_{\hat{j}} \mathcal{M}_{\hat{0} \hat{k} \hat{\ell}}\right)
$$

and

$$
\begin{aligned}
\Phi_{\mathrm{eff}}^{(4)}(t, \vec{r}, \vec{S})= & -\frac{2}{3 m} \mathcal{E}_{\hat{0}} \vec{K} \cdot \vec{\nabla}-\frac{1}{3 m} \vec{\nabla} \cdot\left(\mathcal{E}_{\hat{0}} \vec{K}\right)-\frac{1}{2 m}\left(\mathcal{M}_{\hat{\ell} \hat{j} \hat{0}}+\mathcal{M}_{\hat{0} \hat{j} \hat{\ell}}\right) K^{\hat{\ell}} \frac{\partial}{\partial x_{\hat{j}}}-\frac{1}{4 m} \frac{\partial}{\partial x_{\hat{j}}}\left(\left(\mathcal{M}_{\hat{\ell} \hat{j} \hat{0}}+\mathcal{M}_{\hat{0} \hat{j} \hat{\ell}}\right) K^{\hat{\ell}}\right) \\
& +\frac{2}{3 m} i \vec{S} \cdot \operatorname{rot}\left(\mathcal{E}_{\hat{0}} \vec{K}\right)-\frac{1}{2 m} i \epsilon^{\hat{j} \hat{k} \hat{\ell}} S_{\hat{\ell}} \frac{\partial}{\partial x^{\hat{j}}}\left(\left(\mathcal{M}_{\hat{a} \hat{j} \hat{0}}+\mathcal{M}_{\hat{0} \hat{j} \hat{a}}\right) K^{\hat{a}}\right)
\end{aligned}
$$

and define new torsion-fermion (neutron) low-energy interactions. On the whole the effective low-energy potential Eq.(18) possesses the following properties. First of all, we would like to accentuate that to linear approximation 
in gravitational, chameleon and torsion fields there are no new chameleon-fermion interactions in comparison with those, calculated in [13]. To order $1 / m$ the potential $\Phi_{\mathrm{eff}}^{(3)}(t, \vec{r}, \vec{S})$ describes new torsion-fermion interactions of torsion axial-vector $\overrightarrow{\mathcal{B}}$ and tensor $\mathcal{M}_{\hat{0} \hat{k} \hat{\ell}}$ degrees of freedom. Then, the effective low-energy potential $\Phi_{\text {eff }}^{(4)}(t, \vec{r}, \vec{S})$, containing new torsion interactions with slow fermions to order $1 / m$, is anti-hermitian. It violates invariance under time reversal transformation (or $T$-invariance) and under Charge-Parity transformation (or $C P$-invariance). In section $\amalg$ we discuss in detail such a property of the effective low-energy potential $\Phi_{\mathrm{eff}}^{(4)}(t, \vec{r}, \vec{S})$.

\section{SPACETIME METRIC AND ANTI-HERMITICITY OF THE EFFECTIVE LOW-ENERGY POTENTIAL $\Phi_{\text {eff }}^{(4)}(t, \vec{r}, \vec{S})$}

First of all we would like to note that the metric Eq.(11) is not invariant under time reversal transformation $t \rightarrow-t$. As a result, one can expect a possible violation of $T$-invariance [34]. Since a violation of time reversal invariance is yielded by the effective low-energy potential $\Phi_{\text {eff }}^{(4)}(t, \vec{r}, \vec{S})$, below we analyse step by step the appearance of such a potential.

The operator $\Phi_{\text {eff }}^{(4)}(t, \vec{r}, \vec{S})$ one may obtain from the following terms of the effective low-energy potential Eq.(17)

$$
\begin{aligned}
\delta \Phi_{\mathrm{eff}}(t, \vec{r}, \vec{S}) & =\frac{1}{2 m A} \eta^{\hat{j} \hat{k}} G_{\hat{\jmath}} D_{\hat{k}}^{k} \frac{\partial}{\partial x^{k}}+\frac{1}{4 m} \eta^{\hat{j} \hat{k}} D_{\hat{k}}^{k} \frac{\partial}{\partial x^{k}}\left(\frac{G_{\hat{j}}}{A}\right)-\frac{1}{2 m} i \epsilon^{\hat{\jmath} \hat{\jmath} \hat{\ell}} S_{\hat{\ell}} D_{\hat{k}}^{k} \frac{\partial}{\partial x^{k}}\left(\frac{G_{\hat{j}}}{A}\right) \\
& +\frac{1}{2 m A} \eta^{\hat{j} \hat{k}} G_{\hat{k}} D_{\hat{j}}^{j} \frac{\partial}{\partial x^{j}}+\frac{1}{4 m A} \eta^{\hat{j} \hat{k}} D_{\hat{j}}^{j} \frac{\partial G_{\hat{k}}}{\partial x^{j}}+\frac{1}{2 m A} i \epsilon^{\hat{j} \hat{k} \hat{\ell}} S_{\hat{\ell}} D_{\hat{j}}^{j} \frac{\partial G_{\hat{k}}}{\partial x^{j}} .
\end{aligned}
$$

In the linear approximation for interacting gravitational, chameleon and torsion fields the potential Eq.(23) reduces to the form

$$
\delta \Phi_{\mathrm{eff}}(t, \vec{r}, \vec{S})=-\frac{1}{m} \eta^{\hat{j} \hat{k}} G_{\hat{j}} \frac{\partial}{\partial x^{\hat{k}}}-\frac{1}{2 m} \eta^{\hat{j} \hat{k}} \frac{\partial G_{\hat{j}}}{\partial x^{\hat{k}}}+\frac{1}{m} i \epsilon^{\hat{j} \hat{k} \hat{\ell}} S_{\hat{\ell}} \frac{\partial G_{\hat{k}}}{\partial x^{\hat{j}}}
$$

where we have replaced $A \rightarrow 1, D_{\hat{k}}^{k} \rightarrow-\delta_{\hat{k}}^{k}$ and $D_{\hat{j}}^{j} \rightarrow-\delta_{\hat{j}}^{j}$ (see Eq.(14)). Setting $K^{\hat{j}}=0$ we get (see the Appendix)

$$
G_{\hat{j}}=-\frac{1}{2} \frac{\partial}{\partial x^{\hat{j}}}\left(U_{+}+U_{-}\right) .
$$

The contribution of the operator $G_{\hat{j}}$, given by Eq.(25), is important for the derivation of the gravitational-chameleon part of the effective low-energy potential $\Phi_{\text {eff }}^{(2)}(t, \vec{r}, \vec{S})$, which is Hermitian. This confirms the correctness of the terms proportional to $G_{\hat{j}}$ and $G_{\hat{k}}$ in the effective low-energy potential $\Phi_{\text {eff }}(t, \vec{r}, \vec{S})$.

In the curved spacetime with torsion and metric Eq.(11) the operator $G_{\hat{j}}$ acquires a certain contribution of the torsion field (see the Appendix)

$$
G_{\hat{j}}=-\frac{1}{2} \frac{\partial}{\partial x^{\hat{j}}}\left(U_{+}+U_{-}\right)+\frac{2}{3} \mathcal{E}_{\hat{0}} K_{\hat{j}}+\frac{1}{2}\left(\mathcal{M}_{\hat{\ell} \hat{j} \hat{0}}+\mathcal{M}_{\hat{0} \hat{j} \hat{\ell}}\right) K^{\hat{\ell}} .
$$

Another confirmation of the correctness of the calculation of the contribution of torsion field to the operator $G_{\hat{j}}$ is a cancellation of the part independent of $K^{\hat{j}}$. Indeed, a direct calculation of the torsion $K^{\hat{j}}$-independent part in the operator $G_{\hat{j}}$ gives (see the Appendix)

$$
\left.G_{\hat{j}}\right|_{K^{\hat{j}}=0} ^{\text {torsion }}=\frac{1}{2} \mathcal{T}^{\hat{\alpha}}{ }_{\hat{\alpha} \hat{j}}+\mathcal{K}_{\hat{j} \hat{0} \hat{0}}+\mathcal{K}_{\hat{j} \hat{\ell} \hat{k}} \eta^{\hat{\ell} \hat{k}}=\frac{1}{2} \mathcal{T}_{\hat{0} \hat{0} \hat{j}}+\frac{1}{2} \mathcal{T}_{\hat{j} \hat{\ell} \hat{k}} \eta^{\hat{\ell} \hat{k}}+\frac{1}{2} \mathcal{K}_{\hat{j} \hat{0} \hat{0}}+\frac{1}{2} \mathcal{K}_{\hat{j} \hat{\ell} \hat{k}} \eta^{\hat{\ell} \hat{k}}=0
$$

In the right-hand-side (r.h.s.) of Eq.(27) the first and second terms are cancelled by the third and fourth ones, respectively. This agrees well with results, obtained by Kostelecky [35] (see also [13]). In turn, the contribution of the $K^{\hat{j}}$-dependent part (see the Appendix)

$$
\begin{aligned}
\left.G_{\hat{j}}\right|_{O\left(K^{\hat{j}}\right)} ^{\text {torsion }} & =\frac{1}{2}\left(\mathcal{K}_{\hat{j} \hat{0} \hat{\ell}}+\mathcal{K}_{\hat{j} \hat{\ell} \hat{0}}\right) K^{\hat{\ell}}-\frac{1}{2} K_{\hat{j}} \mathcal{K}_{\hat{0} \hat{\ell} \hat{k}} \eta^{\hat{\ell} \hat{k}}=\frac{1}{2}\left(\mathcal{T}_{\hat{0} \hat{j} \hat{\ell}}+\mathcal{T}_{\hat{\ell} \hat{j} \hat{0}}\right) K^{\hat{\ell}}+\frac{1}{2} K_{\hat{j}} \mathcal{T}_{\hat{\ell} \hat{k} \hat{0} \hat{l}} \eta^{\hat{\ell} \hat{k}}= \\
& =\frac{2}{3} \mathcal{E}_{\hat{0}} K_{\hat{j}}+\frac{1}{2}\left(\mathcal{M}_{\hat{\ell} \hat{j} \hat{0}}+\mathcal{M}_{\hat{0} \hat{j} \hat{\ell}}\right) K^{\hat{\ell}} \neq 0,
\end{aligned}
$$


which is fully correlated with the $K^{\hat{j}}$-independent part through the affine connection (see the Appendix), does not vanish. Hence, taking the operator $G_{\hat{j}}$, given by Eq.(26), we obtain the effective low-energy potential $\Phi_{\text {eff }}^{(4)}(t, \vec{r}, \vec{S})$, which is anti-Hermitian only due to the contribution of torsion and contains both torsion-non-spin-matter and torsionspin-matter interactions. Thus, we have proved that the appearance of the anti-Hermitian potential $\Phi_{\text {eff }}^{(4)}(t, \vec{r}, \vec{S})$ is not a mistake of the calculation but an objective reality, caused by the presence of the torsion scalar $\mathcal{E}_{\hat{0}}$ and tensor $\mathcal{M}_{\hat{\ell} \hat{j} \hat{0}}$ and $\mathcal{M}_{\hat{0} \hat{j} \hat{\ell}}$ degrees of freedom in rotating coordinate systems.

\section{HEISENBERG'S EQUATION FOR SPIN OPERATOR OF SLOW NEUTRONS IN EINSTEIN-CARTAN GRAVITY WITH TORSION, CHAMELEON AND MAGNETIC FIELDS}

A time evolution of the neutron spin operator $\vec{S}$ is described by Heisenberg's equation of motion [36]

$$
\frac{d \vec{S}}{d t}=\frac{\partial \vec{S}}{\partial t}+i[\mathrm{H}, \vec{S}] .
$$

Since the spin operator $\vec{S}$ does not depend explicitly on time, the partial derivative in Eq.(29) is equal to zero. This yields

$$
\frac{d \vec{S}}{d t}=i[\mathrm{H}, \vec{S}]
$$

Since the operator of the kinetic energy of slow neutrons commutes with a neutron spin, we arrive at the equation

$$
\frac{d S^{\hat{a}}}{d t}=\epsilon^{\hat{a} \hat{b} \hat{c}} \Omega_{\mathrm{mb}} S_{\hat{c}}+i\left[\tilde{\Phi}_{\mathrm{eff}}(t, \vec{r}, \vec{S}), S^{\hat{a}}\right]
$$

where $\Omega_{m \hat{b}}=-\kappa_{n} \mu_{N} B_{\hat{b}}$ with $B_{\hat{b}}=(-\vec{B})_{\hat{b}}\left(\right.$ or $\left.\vec{\Omega}_{m}=-\kappa_{n} \mu_{N} \vec{B}\right)$, is the standard angular velocity of the neutron spin precession in the magnetic field $\vec{B}$ [27]. For the calculation of the term $\epsilon^{\hat{a} \hat{b} \hat{c}} \Omega_{m \hat{b}} S_{\hat{c}}=\left(\vec{\Omega}_{m} \times \vec{S}\right)^{\hat{a}}$, where $\Omega_{m \hat{b}}=(-\vec{\Omega} m)_{\hat{b}}$ and $S_{\hat{c}}=(-\vec{S})_{\hat{c}}$, we have used the commutation relation $\left[S^{\hat{b}}, S^{\hat{a}}\right]=i \epsilon^{\hat{a} \hat{b} \hat{c}} S_{\hat{c}}$. The contribution of the commutator $\left[\Phi_{\mathrm{eff}}(t, \vec{r}, \vec{S}), S^{\hat{a}}\right]$ can be written in the following standard form

$$
i\left[\tilde{\Phi}_{\mathrm{eff}}(t, \vec{r}, \vec{S}), S^{\hat{a}}\right]=\epsilon^{\hat{a} \hat{b} \hat{c}} \Omega_{\hat{b}} S_{\hat{c}}=(\vec{\Omega} \times \vec{S})^{\hat{a}},
$$

where $\vec{\Omega}$ is the angular velocity operator of the neutron spin precession, determined by

$$
\vec{\Omega}=\vec{\Omega}_{\mathrm{r}}+\vec{\Omega}_{\mathrm{gr}-\mathrm{ch}}+\vec{\Omega}_{\mathrm{t}}+\vec{\Omega}_{\overline{\mathrm{h}}}
$$

where the indices $\mathrm{r}$, gr $-\mathrm{ch}$, t and $\bar{h}$ mean "rotation","gravitation-chameleon", "torsion" and "anti-Hermitian", respectively. The angular velocity operators in the r.h.s. of Eq. (33) are equal to

$$
\begin{aligned}
\Omega_{\mathrm{r}}^{\hat{b}}= & -\frac{1}{2} \epsilon^{\hat{b} \hat{k} \hat{\ell}} \frac{\partial}{\partial x^{\hat{k}}} K_{\hat{\ell}}, \\
\Omega_{\mathrm{gr}-\mathrm{ch}}^{\hat{b}}= & -\frac{i}{2 m} \epsilon^{\hat{b} \hat{j} \hat{k}} \frac{\partial}{\partial x^{\hat{j}}}\left(U_{+}+2 U_{-}\right) \frac{\partial}{\partial x^{\hat{k}}}, \\
\Omega_{\mathrm{t}}^{\hat{b}}= & -\frac{1}{2} \mathcal{B}^{\hat{b}}-\frac{1}{3} \mathcal{K} K^{\hat{b}}+\frac{1}{2} \epsilon^{\hat{b} \hat{j} \hat{k}} K_{\hat{j}} \mathcal{M}_{\hat{0} \hat{0} \hat{k}}-\frac{1}{2} \epsilon^{\hat{b} \hat{k} \hat{\ell}} \mathcal{M}_{\hat{k} \hat{\ell} \hat{j}} K^{\hat{j}}-\frac{i}{2 m} \mathcal{K} \frac{\partial}{\partial x_{\hat{b}}}-\frac{i}{4 m} \frac{\partial}{\partial x_{\hat{b}}} \mathcal{K} \\
& +\frac{i}{6 m}(\vec{K} \cdot \overrightarrow{\mathcal{B}}) \frac{\partial}{\partial x_{\hat{b}}}+\frac{i}{12 m} \frac{\partial}{\partial x_{\hat{b}}}(\vec{K} \cdot \overrightarrow{\mathcal{B}})+\frac{i}{4 m}\left(\epsilon^{\hat{j} \hat{k} \hat{\ell}} K_{\hat{j}} \mathcal{M}_{\hat{0} \hat{k} \hat{\ell}}\right) \frac{\partial}{\partial x_{\hat{b}}}+\frac{i}{8 m} \frac{\partial}{\partial x_{\hat{b}}}\left(\epsilon^{\hat{j} \hat{k} \hat{\ell}} K_{\hat{j}} \mathcal{M}_{\hat{0} \hat{k} \hat{\ell}}\right), \\
\Omega_{\hat{\mathrm{h}}}^{\hat{b}}= & -\frac{2}{3 m} i \epsilon^{\hat{\epsilon} \hat{k} \hat{\ell}} \frac{\partial}{\partial x^{\hat{k}}}\left(\mathcal{E}_{\hat{0}} K_{\hat{\ell}}\right)+\frac{1}{2 m} i \epsilon^{\hat{b} \hat{k} \hat{\ell}} \frac{\partial}{\partial x^{\hat{\ell}}}\left(\left(\mathcal{M}_{\hat{j} \hat{k} \hat{0}}+\mathcal{M}_{\hat{0} \hat{k} \hat{j}}\right) K^{\hat{j}}\right) .
\end{aligned}
$$


For the coordinate system, rotating with an angular velocity $\vec{\omega}$, where $K^{\hat{j}}=-\epsilon^{\hat{j} \hat{k} \hat{\ell}} \omega_{\hat{k}} x_{\hat{\ell}}=-(\vec{\omega} \times \vec{r})^{\hat{j}}$, we get

$$
\begin{aligned}
\Omega_{\mathrm{r}}^{\hat{b}}= & -\omega^{\hat{b}}, \\
\Omega_{\mathrm{gr}-\mathrm{ch}}^{\hat{b}}= & -i \frac{3}{2 m}\left(\vec{\nabla} U_{\mathrm{E}} \times \vec{\nabla}\right)^{\hat{b}}+i \frac{1}{2 m} \frac{\beta}{M_{\mathrm{PI}}}(\vec{\nabla} \phi \times \vec{\nabla})^{\hat{b}}, \\
\Omega_{\mathrm{t}}^{\hat{b}}= & -\frac{1}{2} \mathcal{B}^{\hat{b}}+\frac{1}{3}(\vec{\omega} \times \vec{r} \mathcal{K})^{\hat{b}}-\frac{1}{2}\left(x^{\hat{b}}\left(\omega^{\hat{k}} \mathcal{M}_{\hat{0} \hat{0} \hat{k}}\right)-\omega^{\hat{b}}\left(x^{\hat{k}} \mathcal{M}_{\hat{0} \hat{0} \hat{k}}\right)\right)+\frac{1}{2} \epsilon^{\hat{b} \hat{k} \hat{\ell}} \mathcal{M}_{\hat{k} \hat{\ell} \hat{j}} \epsilon^{\hat{j} \hat{p} \hat{q}} \omega_{\hat{p}} x_{\hat{q}} \\
& -\frac{i}{2 m} \mathcal{K} \frac{\partial}{\partial x_{\hat{b}}}-\frac{i}{4 m} \frac{\partial}{\partial x_{\hat{b}}} \mathcal{K}-\frac{i}{6 m}(\vec{\omega} \cdot(\vec{r} \times \overrightarrow{\mathcal{B}})) \frac{\partial}{\partial x_{\hat{b}}}-\frac{i}{12 m} \frac{\partial}{\partial x_{\hat{b}}}(\vec{\omega} \cdot(\vec{r} \times \overrightarrow{\mathcal{B}})) \\
& +\frac{i}{4 m}\left(\left(\omega^{\hat{k}} x^{\hat{\ell}}-\omega^{\hat{\ell}} x^{\hat{k}}\right) \mathcal{M}_{\hat{0} \hat{k} \hat{\ell}}\right) \frac{\partial}{\partial x_{\hat{b}}}+\frac{i}{8 m} \frac{\partial}{\partial x_{\hat{b}}}\left(\left(\omega^{\hat{k}} x^{\hat{\ell}}-\omega^{\hat{\ell}} x^{\hat{k}}\right) \mathcal{M}_{\hat{0} \hat{k} \hat{\ell}}\right), \\
\Omega_{\frac{\mathrm{h}}{\hat{b}}=} & -\frac{2}{3 m} i\left(\vec{\nabla} \mathcal{E}_{\hat{0}} \times(\vec{\omega} \times \vec{r})+2 \mathcal{E}_{\hat{0}} \vec{\omega}\right)^{\hat{b}}+\frac{1}{2 m} i \epsilon^{\hat{b} \hat{k} \hat{\ell}} \frac{\partial}{\partial x^{\hat{\ell}}}\left(\left(\mathcal{M}_{\hat{j} \hat{k} \hat{0}}+\mathcal{M}_{\hat{0} \hat{k} \hat{j}}\right) \epsilon^{\hat{j} \hat{p} \hat{q}} \omega_{\hat{p}} x_{\hat{q}}\right) .
\end{aligned}
$$

The experimental analysis of the spin-rotation couplings, calculated above, can be, for example, carried out by neutron interferometer [37]- 49] with rotating interferometers [2, 3, 49].

\section{PROPERTIES OF THE DIRAC HAMILTON OPERATOR IN CURVED SPACETIMES}

The anti-Hermitian interactions appears also in the Hamilton operator of a relativistic Dirac fermion with mass $m$. Indeed, as has been shown in [1] the Hamilton operator of a relativistic Dirac fermion is equal to $\mathrm{H}^{\prime}=\mathrm{H}_{0}+\delta \mathrm{H}^{\prime}$, where $\mathrm{H}_{0}=\gamma^{\hat{0}} m-i \gamma^{\hat{0}} \vec{\gamma} \cdot \vec{\nabla}$ is the Hamilton operator of a free Dirac fermion with mass $m$ and $\delta \mathrm{H}^{\prime}$ is the interaction Hamilton operator equal to [1]

$$
\delta \mathrm{H}^{\prime}=(A-1) \gamma^{\hat{0}} m+B+C^{\hat{\ell}} \Sigma_{\hat{\ell}}+\left(D_{\hat{j}}^{j}+\delta_{\hat{j}}^{j}\right) i \gamma^{\hat{0}} \gamma^{\hat{j}} \frac{\partial}{\partial x^{j}}+F_{\hat{j}} i \gamma^{\hat{0}} \gamma^{\hat{j}} \frac{\partial}{\partial t}+G_{\hat{j}} i \gamma^{\hat{0}} \gamma^{\hat{j}}+K \gamma^{5}+L^{\hat{j}} i \frac{\partial}{\partial x^{\hat{j}}}
$$

For the operators $A, B, C^{\hat{\ell}}, D^{j} \hat{j}, G_{\hat{j}}, K$ and $L^{\hat{j}}$, given by Eq. (14), the Hamilton operator can be represented in the form $\delta \mathrm{H}^{\prime}=\delta \mathrm{H}_{\mathrm{h}}^{\prime}+\delta \mathrm{H}_{\overline{\mathrm{h}}}^{\prime}$, where $\delta \mathrm{H}_{\mathrm{h}}^{\prime}$ and $\delta \mathrm{H}_{\overline{\mathrm{h}}}^{\prime}$ are the Hermitian and anti-Hermitian parts of the Hamilton operator Eq.(36), respectively, equal to

$$
\begin{aligned}
\delta \mathrm{H}_{\mathrm{h}}^{\prime} & =U_{+} \gamma^{\hat{0}} m-\left(U_{+}+U_{-}\right) i \gamma^{\hat{0}} \vec{\gamma} \cdot \vec{\nabla}-\frac{1}{2} i \gamma^{\hat{0}} \vec{\nabla}\left(U_{+}+U_{-}\right) \\
& -\frac{1}{2} i \operatorname{div} \vec{K}-i \vec{K} \cdot \vec{\nabla}+\frac{1}{4} \vec{\Sigma} \cdot \operatorname{rot} \vec{K} \\
& -\frac{1}{4} \vec{\Sigma} \cdot \overrightarrow{\mathcal{B}}-\frac{1}{6} \mathcal{K} \vec{\Sigma} \cdot \vec{K}+\frac{1}{4} \epsilon^{\hat{\ell} \hat{j} \hat{k}} \Sigma_{\hat{\ell}} K_{\hat{j}} \mathcal{M}_{\hat{0} \hat{0} \hat{k}}+\frac{1}{4} \epsilon^{\hat{\ell} \hat{j} \hat{k}} \Sigma_{\hat{\ell}} \mathcal{M}_{\hat{j} \hat{k} \hat{a}} K^{\hat{a}} \\
& -\frac{1}{4} \mathcal{K} \gamma^{5}+\frac{1}{12} \vec{K} \cdot \overrightarrow{\mathcal{B}} \gamma^{5}+\frac{1}{8} \epsilon^{\hat{j} \hat{k} \hat{\ell}} K_{\hat{j}} \mathcal{M}_{\hat{0} \hat{k} \hat{\ell}} \gamma^{5}
\end{aligned}
$$

and

$$
\delta \mathrm{H}_{\overline{\mathrm{h}}}^{\prime}=-i \frac{2}{3} \mathcal{E}_{\hat{0}} \vec{K} \cdot \gamma^{\hat{0}} \vec{\gamma}+i \frac{1}{2}\left(\mathcal{M}_{\hat{\ell} \hat{j} \hat{0}}+\mathcal{M}_{\hat{0} \hat{j} \hat{\ell}}\right) K^{\hat{\ell}} \gamma^{\hat{0}} \gamma^{\hat{j}}
$$

One may assume that in the rotating Universe and galaxies 50] (see also [51]) the torsion-fermion interaction Eq.(38) might be an origin of i) violation of $C P$ and $T$ invariance in the Universe and ii) of baryon asymmetry [28].

\section{A. Standard non-unitary transformation of Dirac fermion wave functions and anti-Hermitian torsion-fermion interactions}

It is well-known that the Hamilton operator of the Dirac fermions with mass $m$, moving in the curved spacetime with a metric $g_{\mu \nu}$, is not Hermitian. In order to get a Hermitian Hamilton operator one has to perform the standard non-unitary transformation of the wave function of the Dirac fermions $\psi \rightarrow\left(\sqrt{-g} e_{0}^{0}\right)^{1 / 2} \psi^{\prime}$, where $g$ is a determinant of the metric tensor $g_{\mu \nu}(x)$ and $e_{0}^{0}(x)$ is a vierbein field [17, 18], 14, 15, 19], 16], 20, 21] and [24] (see also [1, 13, 22, 23]). 
As has been shown in [1], the Hamilton operator $\mathrm{H}^{\prime}=\gamma^{\hat{0}} m-i \gamma^{\hat{0}} \vec{\gamma} \cdot \vec{\nabla}+\delta \mathrm{H}_{\mathrm{h}}^{\prime}+\delta \mathrm{H}_{\overline{\mathrm{h}}}^{\prime}$ has been already obtained by means of the standard non-unitary transformation $\psi \rightarrow\left(\sqrt{-\tilde{g}} \tilde{e}_{0}^{\hat{0}}\right)^{1 / 2} \psi^{\prime}$, where $\tilde{g}$ is a determinant of the Jordan-frame metric tensor $\tilde{g}_{\mu \nu}(x)$ and $\tilde{e}_{0}^{\hat{0}}(x)$ is a vierbein field in the Jordan frame (see Eq. (13) of Ref. [1] ). The appearance of the anti-Hermitian term $\delta \mathrm{H}_{\overline{\mathrm{h}}}^{\prime}$ in the Hamilton operator $\mathrm{H}^{\prime}=\gamma^{\hat{0}} m-i \gamma^{\hat{0}} \vec{\gamma} \cdot \vec{\nabla}+\delta \mathrm{H}_{\mathrm{h}}^{\prime}+\delta \mathrm{H}_{\overline{\mathrm{h}}}^{\prime}$ is fully related to the spacetime metric Eq. (11) as a functional of the vector $\vec{K}$, caused by rotations, or more generally to the spacetime metric Eq.(3) (see also Eq.(20) of Ref.[1]), proposed by Obukhov, Silenko, and Teryaev [14 16].

\section{B. Non-unitary transformations of Dirac fermion wave functions and removal of anti-Hermitian torsion-fermion interactions}

Now we would like to show that the anti-Hermitian (non-Hermitian) Hamilton operator $\delta \mathrm{H}_{\overline{\mathrm{h}}}^{\prime}$, given by Eq. (38), cannot be removed by a non-unitary (non-Hermitian) transformation of the Dirac fermion (neutron) wave function. After the standard non-unitary transformation of the Dirac fermion wave function $\psi \rightarrow\left(\sqrt{-\tilde{g}} \tilde{e}_{0}^{\hat{0}}\right)^{1 / 2} \psi^{\prime}$ (see Eq. (13) of Ref.[1]) we arrive at the following Dirac fermion action

$$
S_{\psi}=\int d t d^{3} x \psi^{\prime \dagger}(t, \vec{r})\left(i \frac{\partial}{\partial t}-\mathrm{H}^{\prime}\right) \psi^{\prime}(t, \vec{r})=\int d t d^{3} x \psi^{\prime \dagger}(t, \vec{r})\left(i \frac{\partial}{\partial t}-\mathrm{H}_{0}-\delta \mathrm{H}_{\mathrm{h}}^{\prime}-\delta \mathrm{H}_{\overline{\mathrm{h}}}^{\prime}\right) \psi^{\prime}(t, \vec{r}),
$$

where $\mathrm{H}_{0}=\gamma^{\hat{0}} m-i \gamma^{\hat{0}} \vec{\gamma} \cdot \vec{\nabla}$. In order to analyse a possibility to remove the term $\delta \mathrm{H}_{\overline{\mathrm{h}}}^{\prime}$ we make a non-unitary (non-Hermitian) transformation

$$
\psi^{\prime}(t, \vec{r})=\zeta \psi^{\prime \prime}(t, \vec{r})
$$

where $\zeta=1+Q=1+\eta_{\hat{j} \hat{k}} O^{\hat{j}} \gamma^{\hat{k}}$ and $O^{\hat{j}}$ is a Hermitian non-differential operator, i.e. $Q^{\hat{j} \dagger}=Q^{\hat{j}}$. The operator $\zeta$ is a non-unitary (non-Hermitian) operator $\zeta^{\dagger}=1+Q^{\dagger}=1-\eta_{\hat{j} \hat{k}} O^{\hat{j}} \gamma^{\hat{k}} \neq \zeta$. Then, for the derivation of the Dirac Hamilton operator $\mathrm{H}^{\prime \prime}$ we use the following relations $\zeta^{\dagger} \delta \mathrm{H}_{\mathrm{h}}^{\prime}=\delta \mathrm{H}_{\mathrm{h}}^{\prime}$ and of $\zeta^{\dagger} \delta \mathrm{H}_{\overline{\mathrm{h}}}^{\prime}=\delta \mathrm{H}_{\overline{\mathrm{h}}}^{\prime}$ and $\delta \mathrm{H}_{\mathrm{h}}^{\prime} \zeta=\delta \mathrm{H}_{\mathrm{h}}^{\prime}$ and $\delta \mathrm{H}_{\overline{\mathrm{h}}}^{\prime} \zeta=\delta \mathrm{H}_{\overline{\mathrm{h}}}^{\prime}$. Plugging Eq.(40) into Eq.(39) we transcribe the action $S_{\psi}$ into the form

$$
S_{\psi}=\int d t d^{3} x \psi^{\prime \prime \dagger}(t, \vec{r})\left(i \frac{\partial}{\partial t}-\mathrm{H}^{\prime \prime}\right) \psi^{\prime \prime}(t, \vec{r}),
$$

where the Hamilton operator $\mathrm{H}^{\prime \prime}$ is equal to

$$
\mathrm{H}^{\prime \prime}=\mathrm{H}_{0}+\delta \mathrm{H}_{\mathrm{h}}^{\prime}+\delta \mathrm{H}_{\overline{\mathrm{h}}}^{\prime}+\left[\mathrm{H}_{0}, Q\right]-i \frac{\partial Q}{\partial t} .
$$

Plugging $Q=\eta_{\hat{j} \hat{k}} O^{\hat{j}} \gamma^{\hat{k}}$ into Eq. (42) and calculating the commutator $\left[\mathrm{H}_{0}, Q\right]$ we get

$$
\mathrm{H}^{\prime \prime}=\mathrm{H}_{0}+\delta \mathrm{H}_{\mathrm{h}}^{\prime}+\delta \mathrm{H}_{\overline{\mathrm{h}}}^{\prime}+2 m \eta_{\hat{j} \hat{k}} Q^{\hat{j}} \gamma^{\hat{0}} \gamma^{\hat{k}}-i \gamma^{\hat{0}} \frac{\partial Q^{\hat{j}}}{\partial x^{\hat{j}}}-2 i \gamma^{\hat{0}} Q^{\hat{j}} \frac{\partial}{\partial x^{\hat{j}}}+\varepsilon^{\hat{j} \hat{k} \hat{k}} \gamma^{\hat{0}} \Sigma_{\hat{j}} \frac{\partial Q_{\hat{k}}}{\partial x^{\hat{\ell}}}-i \frac{\partial Q_{\hat{j}}}{\partial t} \gamma^{\hat{j}} .
$$

It is obvious that for the Hermitian operator $Q^{\hat{j}}$, i.e. $Q^{\hat{j} \dagger}=Q^{\hat{j}}$ corresponding to a non-Hermitian transformation with the operator $\zeta^{\dagger} \neq \zeta$, the term $2 m \eta_{\hat{j} \hat{k}} Q^{\hat{j}} \gamma^{\hat{0}} \gamma^{\hat{k}}$ cannot cancel the contribution of the anti-Hermitian operator $\delta \mathrm{H}_{\overline{\mathrm{h}}}^{\prime}$. The use of the anti-Hermitian operator $Q^{\hat{j}} \rightarrow i Q^{\hat{j}}$, corresponding to a Hermitian (unitary) transformation with an operator $\zeta=1+i Q=1+i \eta_{\hat{j} \hat{k}} O^{\hat{j}} \gamma^{\hat{k}}$ such as $\zeta^{\dagger}=\zeta$, allows to shift the Hamilton operator $\delta \mathrm{H}_{\overline{\mathrm{h}}}^{\prime}$, which is the odd operator according to the Foldy-Wouthuysen classification [52], to the region of interactions of order $O(1 / m)$. In detail such a unitary transformation or the Foldy-Wouthuysen transformation for the derivation of the effective low-energy potential Eq. (17) has been performed in [1]. The linearised version of this effective low-energy potential is given by Eq.(18).

\section{Non-Hermiticity of Dirac Hamilton operator, $\eta$-representation and anti-Hermitian torsion-fermion interactions}

An alternative transition from a non-Hermitian Hamilton operator of the Dirac massive fermions, moving in the curved spacetime with an arbitrary metric tensor $g_{\mu \nu}(x)$, to a Hermitian form can be performed by using the $\eta-$ representation of the Dirac fermion wave functions [24]. In the $\eta$-representation the Dirac Hamilton operator becomes 
Hermitian without the standard non-unitary transformation of the Dirac fermion wave function $\psi \rightarrow\left(\sqrt{-\tilde{g}} \tilde{e}_{\hat{0}}^{0}\right)^{1 / 2} \psi^{\prime}$ and the dynamics of the Dirac fermions is described by the pseudo-Hermitian quantum mechanics [24] (see also [5355]). Since in our analysis of the torsion-fermion interactions within the Einstein-Cartan gravity with the chameleon field we use the standard non-unitary transformation of the Dirac fermion wave function $\psi \rightarrow\left(\sqrt{-\tilde{g}} \tilde{e}_{\hat{0}}^{0}\right)^{1 / 2} \psi^{\prime}$, the dynamics of the Dirac fermions and the contributions of the anti-Hermitian torsion-fermion interactions, violating $C P$ and $T$ invariance, to the observables can be described within the formalism of the standard relativistic and non-relativistic quantum mechanics [56, 57].

\section{Conformal invariance of anti-Hermitian torsion-fermion interactions}

As has been shown by Silenko [20], the Dirac and Foldy-Wouthuysen Hamilton operators for massless fermions in the curved spacetimes with arbitrary metric $\tilde{g}_{\mu \nu}$ are invariant under conformal transformation $\tilde{g}_{\mu \nu} \rightarrow O^{2} \check{g}_{\mu \nu}$ if the wave function of massless fermions is subjected to the non-unitary transformation $\psi^{\prime} \rightarrow O^{3 / 2} \check{\psi}$. In $[21]$ the results, obtained in [20], have been extended to massive fermions coupled to gravitational field and torsion in the Einstein and Einstein-Cartan gravity, respectively, with the requirement that the fermion mass transforms under the conformal transformation $\tilde{g}_{\mu \nu} \rightarrow O^{2} \check{g}_{\mu \nu}$ as follows $m \rightarrow O^{-1} \check{m}$. This agrees well with the dimensional analysis of general relativity, carried out by Dicke [26] for the reduction of the Brans-Dicke gravitational theory [25] to the Einstein gravity, coupled to an effective scalar field. According to Silenko 21], the vector $K^{j}$ and the contorsion tensor $\tilde{\mathcal{K}}_{\alpha \mu \nu}=-\frac{1}{2}\left(\tilde{\mathcal{T}}_{\alpha \mu \nu}-\tilde{\mathcal{T}}_{\mu \alpha \nu}-\tilde{\mathcal{T}}_{\nu \alpha \mu}\right)$ are not changed by the conformal transformation $\tilde{g}_{\mu \nu} \rightarrow O^{2} \check{g}_{\mu \nu}$, i.e. $K^{j} \rightarrow \check{K}^{j}=K^{j}$ and $\tilde{\mathcal{K}}_{\alpha \mu \nu} \rightarrow \check{\mathcal{K}}_{\alpha \mu \nu}$. Since the Hamilton operator $\delta \mathrm{H}_{\hat{h}}$ is expressed in terms of the components of the contorsion tensor $\mathcal{K}_{\hat{\alpha} \hat{\mu} \hat{\nu}}$ and the vector $K^{\hat{j}}$

$$
\begin{aligned}
\delta \mathrm{H}_{\overline{\mathrm{h}}}^{\prime} & =\left.G_{\hat{j}}\right|_{O\left(K^{\hat{j}}\right)} ^{\text {torsion }} i \gamma^{\hat{0}} \gamma^{\hat{j}}=\frac{1}{2}\left(\mathcal{K}_{\hat{j} \hat{0} \hat{\ell}}+\mathcal{K}_{\hat{j} \hat{\ell} \hat{0}}\right) K^{\hat{\ell}} i \gamma^{\hat{0}} \gamma^{\hat{j}}-\frac{1}{2} K_{\hat{j}} \mathcal{K}_{\hat{0} \hat{\ell} \hat{k}} \eta^{\hat{\ell} \hat{k}} i \gamma^{\hat{0}} \gamma^{\hat{j}}= \\
& =-i \frac{2}{3} \mathcal{E}_{\hat{0}} \vec{K} \cdot \gamma^{\hat{0}} \vec{\gamma}+i \frac{1}{2}\left(\mathcal{M}_{\hat{\ell} \hat{j} \hat{0}}+\mathcal{M}_{\hat{0} \hat{j} \hat{\ell}}\right) K^{\hat{\ell}} \gamma^{\hat{0}} \gamma^{\hat{j}},
\end{aligned}
$$

it is invariant under the conformal transformation $\tilde{g}_{\mu \nu} \rightarrow O^{2} \check{g}_{\mu \nu}$, i.e. $\delta \mathrm{H}_{\overline{\mathrm{h}}}^{\prime} \rightarrow \delta \check{\mathrm{H}}_{\overline{\mathrm{h}}}^{\prime}$, where $\left.G_{\hat{j}}\right|_{O\left(K^{\hat{j}}\right)} ^{\text {torsion }}$, given by (see $\mathrm{Eq} \cdot(\underline{28}))$

$$
\left.G_{\hat{j}}\right|_{O\left(K^{\hat{j}}\right)} ^{\text {torsion }}=\frac{1}{2}\left(\mathcal{K}_{\hat{j} \hat{0} \hat{\ell}}+\mathcal{K}_{\hat{j} \hat{\ell} \hat{0}}\right) K^{\hat{\ell}}-\frac{1}{2} K_{\hat{j}} \mathcal{K}_{\hat{0} \hat{\ell} \hat{k}} \eta^{\hat{\ell} \hat{k}}
$$

is conformal invariant $\left.\left.G_{\hat{j}}\right|_{O\left(K^{\hat{j}}\right)} ^{\text {torsion }} \rightarrow \check{G}_{\hat{j}}\right|_{O\left(K^{\hat{j}}\right)} ^{\text {torsion }}$. Under the conformal transformation $\tilde{g}_{\mu \nu} \rightarrow O^{2} \check{g}_{\mu \nu}$ the vierbein fields transform as follows $\tilde{e}_{\mu}^{\hat{\alpha}} \rightarrow O \check{e}_{\mu}^{\hat{\alpha}}$ and $\tilde{e}_{\hat{\alpha}}^{\mu} \rightarrow O^{-1} \check{e}_{\hat{\alpha}}^{\mu}$. Since the fermion mass transforms as $m \rightarrow O^{-1} \check{m}$, the operators $m A=-m \tilde{e}_{0}^{\hat{0}}$ and $D_{\hat{j}}^{j}=-\tilde{e}_{0}^{\hat{0}} \tilde{e}_{\hat{j}}^{j}$ (see Eq.(17)) are invariant under the conformal transformation, i.e. $m A \rightarrow \check{m} \check{A}$ and $D_{\hat{j}}^{j} \rightarrow \check{D}_{\hat{j}}^{j}$. In order to show that the effective low-energy potential $\Phi_{\mathrm{eff}}^{(4)}(t, \vec{r}, \vec{S})$ is conformal invariant we transcribe the effective low-energy potential $\delta \Phi_{\mathrm{eff}}(t, \vec{r}, \vec{S})$, given by Eq.(23), into the form

$$
\begin{aligned}
\delta \Phi_{\mathrm{eff}}^{\text {torsion }}(t, \vec{r}, \vec{S}) & =\left.\frac{1}{2 m A} \eta^{\hat{j} \hat{k}} G_{\hat{j}}\right|_{O\left(K^{\hat{j}}\right)} ^{\text {torsion }} D_{\hat{k}}^{k} \frac{\partial}{\partial x^{k}}+\eta^{\hat{j} \hat{k}} D_{\hat{k}}^{k} \frac{\partial}{\partial x^{k}}\left(\left.\frac{1}{4 m A} G_{\hat{j}}\right|_{O\left(K^{\hat{j}}\right)} ^{\text {torsion }}\right)-i \epsilon^{\hat{j} \hat{k} \hat{\ell}} S_{\hat{\ell}} D_{\hat{k}}^{k} \frac{\partial}{\partial x^{k}}\left(\left.\frac{1}{2 m A} G_{\hat{j}}\right|_{O\left(K^{\hat{j}}\right)} ^{\text {torsion }}\right) \\
& +\left.\frac{1}{2 m A} \eta^{\hat{j} \hat{k}} G_{\hat{k}}\right|_{O\left(K^{\hat{j}}\right)} ^{\text {torsion }} D_{\hat{j}}^{j} \frac{\partial}{\partial x^{j}}+\left.\frac{1}{4 m A} \eta^{\hat{j} \hat{k}} D_{\hat{j}}^{j} \frac{\partial}{\partial x^{j}} G_{\hat{k}}\right|_{O\left(K^{\hat{j}}\right)} ^{\text {torsion }}+\left.\frac{1}{2 m A} i \epsilon^{\hat{j} \hat{k} \hat{\ell}} S_{\hat{\ell}} D_{\hat{j}}^{j} \frac{\partial}{\partial x^{j}} G_{\hat{k}}\right|_{O\left(K^{\hat{j}}\right)} ^{\text {torsion }}, \quad(46)
\end{aligned}
$$

Because of the conformal invariance of $m A \rightarrow \check{m} \check{A}, D_{\hat{j}}^{j} \rightarrow \check{D}_{\hat{j}}^{j}$ and $\left.\left.G_{\hat{j}}\right|_{O\left(K^{\hat{j}}\right)} ^{\text {torsion }} \rightarrow \check{G}_{\hat{j}}\right|_{O\left(K^{\hat{j}}\right)} ^{\text {torsion }}$ the effective low-energy potential Eq.(46) is conformal invariant, i.e. $\delta \Phi_{\mathrm{eff}}^{\mathrm{torsion}}(t, \vec{r}, \vec{S}) \rightarrow \delta \check{\Phi}_{\mathrm{eff}}^{\mathrm{torsion}}(t, \vec{r}, \vec{S})$. This proves the conformal invariance of the effective low-energy potential $\Phi_{\mathrm{eff}}^{(4)}(t, \vec{r}, \vec{S})$, i.e. $\Phi_{\mathrm{eff}}^{(4)}(t, \vec{r}, \vec{S}) \rightarrow \check{\Phi}_{\mathrm{eff}}^{(4)}(t, \vec{r}, \vec{S})$. Thus, we have shown that after the non-unitary transformation of the Dirac fermion wave function $\psi \rightarrow\left(\sqrt{-\tilde{g}} \tilde{e}_{\hat{0}}^{0}\right)^{1 / 2} \psi^{\prime}$ in the Jordan frame with the metric tensor $\tilde{g}_{\mu \nu}(x)$ the non-Hermitian (anti-Hermitian) torsion-fermion interactions, violating $T$ and $C P$ invariance, are conformal invariant. This agrees well with the results, obtained by Silenko in Ref. [21]. 


\section{E. The argument in behalf of observability of anti-Hermitian torsion-fermion interactions}

Now we would like to discuss a possible observability of the anti-Hermitian torsion-fermion interactions, violating $C P$ and $T$ invariance. For the coordinate system, rotating with an angular velocity $\vec{\omega}$, the vector $\vec{K}$ is equal to $\vec{K}=-(\vec{\omega} \times \vec{r})[14,15,33]$. The effective low-energy potential Eq.(18) is equal to

$$
\tilde{\Phi}_{\mathrm{eff}}(t, \vec{r}, \vec{S})=\left.\Phi_{\mathrm{eff}}^{(1)}(t, \vec{r}, \vec{S})\right|_{\vec{K}=-(\vec{\omega} \times \vec{r})}+\left.\Phi_{\mathrm{eff}}^{(2)}(t, \vec{r}, \vec{S})\right|_{\vec{K}=-(\vec{\omega} \times \vec{r})}+\left.\Phi_{\mathrm{eff}}^{(3)}(t, \vec{r}, \vec{S})\right|_{\vec{K}=-(\vec{\omega} \times \vec{r})}+\left.\Phi_{\mathrm{eff}}^{(4)}(t, \vec{r}, \vec{S})\right|_{\vec{K}=-(\vec{\omega} \times \vec{r})} .
$$

Here the effective low-energy potential $\left.\Phi_{\text {eff }}^{(1)}(t, \vec{r}, \vec{S})\right|_{\vec{K}=-(\vec{\omega} \times \vec{r})}$ is

$$
\begin{aligned}
\left.\Phi_{\mathrm{eff}}^{(1)}(t, \vec{r}, \vec{S})\right|_{\vec{K}=-(\vec{\omega} \times \vec{r})} & =m\left(U_{+}-U_{\mathrm{E}}\right)-\vec{\omega} \cdot \vec{L}-\vec{\omega} \cdot \vec{S}-\frac{1}{2} \vec{S} \cdot \overrightarrow{\mathcal{B}}+\frac{1}{3} \mathcal{K} \vec{S} \cdot(\vec{\omega} \times \vec{r}) \\
& -\frac{1}{2} \vec{S} \cdot(\overrightarrow{\mathcal{M}} \times(\vec{\omega} \times \vec{r}))-\frac{1}{2} S_{j} \epsilon^{j k \ell} \mathcal{M}_{k \ell a} \epsilon^{a b c} \omega_{b} x_{c} .
\end{aligned}
$$

where $(\overrightarrow{\mathcal{M}})_{k}=-\mathcal{M}_{00 k}$ [1]. In Eq.(48) the first term $m\left(U_{+}-U_{\mathrm{E}}\right)$ describes the chameleon-matter interaction [58, 59], whereas the terms $-\vec{\omega} \cdot \vec{L}$ and $-\vec{\omega} \cdot \vec{S}$, where $\vec{L}=-\vec{r} \times i \vec{\nabla}$ is the orbital momentum operator of slow fermions (neutrons), agree well with the results, obtained by Hehl and Ni 33. The interactions $-\vec{\omega} \cdot \vec{L}$ and $-\vec{\omega} \cdot \vec{S}$ were investigated and observed in the experiments by Werner, Staudenmann, and Colella [60], by Atwood et al. [2] and by Mashhoon [3]. Since the effective low-energy potentials $\left.\Phi_{\mathrm{eff}}^{(n)}(t, \vec{r}, \vec{S})\right|_{\vec{K}=-(\vec{\omega} \times \vec{r})}$ for $n=2,3,4$, are calculated from the general effective low-energy potential Eq.(6) as well as the potential $\left.\Phi_{\text {eff }}^{(1)}(t, \vec{r}, \vec{S})\right|_{\vec{K}=-(\vec{\omega} \times \vec{r})}$, the observability of the interactions $-\vec{\omega} \cdot(\vec{L}+\vec{S})$ supports in principle an observability of the torsion-fermion interactions in the effective low-energy potential Eq.(48), the Hermitian interactions $\left.\Phi_{\mathrm{eff}}^{(2)}(t, \vec{r}, \vec{S})\right|_{\vec{K}=-(\vec{\omega} \times \vec{r})}$ and $\left.\Phi_{\mathrm{eff}}^{(3)}(t, \vec{r}, \vec{S})\right|_{\vec{K}=-(\vec{\omega} \times \vec{r})}$ and, correspondingly, the anti-Hermitian interaction $\left.\Phi_{\mathrm{eff}}^{(4)}(t, \vec{r}, \vec{S})\right|_{\vec{K}=-(\vec{\omega} \times \vec{r})}$, respectively.

\section{CONCLUSION}

We have derived the operator of the angular velocity of the neutron spin precession in the Einstein-Cartan gravity with torsion and chameleon fields. For the calculation of such an operator we have used the most general effective low-energy potential for slow Dirac fermions, coupled to gravitational, chameleon and torsion fields to order $1 / m$, where $m$ is the fermion mass [1]. In order to adapt such an effective low-energy potential to the experimental analysis of the contributions of fermion-gravitational,--chameleon and -torsion interactions we have linearised it with respect to gravitational, chameleon and torsion fields. Such a linearisation we have carried out in the curved spacetime with the Schwarzschild metric, taken in the approximation of the weak gravitational field and modified by a rotation with an angular velocity $\vec{\omega}$ and the chameleon field. We have shown that in the curved spacetime with such a modified metric torsion scalar, pseudoscalar, axial-vector and tensor degrees of freedom couple to slow neutrons through minimal torsion-fermion couplings [1]. The obtained linearised effective low-energy potential Eq. (18) is the generalization of the effective low-energy potential, derived in [13].

An important peculiarity of the linearised effective low-energy potential Eq.(18) is the appearance of the antiHermitian part. Such a part of the effective low-energy potential comes from the operator $G_{\hat{j}}$ and proportional to the vector $K^{\hat{j}}=-(\vec{\omega} \times \vec{r})^{\hat{j}}$, related to a rotation of a coordinate system. A possible violation of Hermiticity of a low-energy potential for slow fermions, coupled to gravitational, chameleon and torsion fields in terms of the vierbein fields Eq.(4), caused by the metric Eq.(3), might be expected because of a non-invariance of the metric tensor Eq.(3) and, correspondingly, Eq.(13) in a rotating coordinate system with respect to time reversal transformation, i.e. a noninvariance under $t \rightarrow-t$ transformation. We have found that the anti-Hermitian part contains the terms proportional to vector $K^{\hat{j}}$ and dependent on the torsion scalar $\mathcal{E}_{\hat{0}}$ and space-space-time $\mathcal{M}_{\hat{j} \hat{k} \hat{0}}$ and time-space-space $\mathcal{M}_{\hat{0} \hat{k} \hat{j}}$ tensor degrees of freedom. For the confirmation of the correctness of such an anti-Hermitian part we have pointed out that the contribution of the operator $G_{\hat{j}}$ is important for the correct derivation of the effective low-energy potential for slow fermions (neutrons), coupled to gravitational and chameleon fields. Another argument on behalf of the correctness of such an anti-Hermitian part is the cancellation of the torsion vector components in the $K_{\hat{j}}$-independent part of 
the operator $G_{\hat{j}}$. Such a cancellation agrees well with the results, obtained by Kostelecky [35] (see also [13]). In the Appendix we have given a detailed calculation of the operator $G_{\hat{j}}$ to linear order in the gravitational, chameleon and torsion field approximation.

It is obvious that an anti-Hermitian part $\Phi_{\text {eff }}^{(4)}(t, \vec{r}, \vec{S})$ of the effective low-energy potential $\Phi_{\text {eff }}(t, \vec{r}, \vec{S})$ violates T (or time reversal) invariance. Since the effective low-energy potential $\Phi_{\mathrm{eff}}(t, \vec{r}, \vec{S})$ is invariant under CPT transformation, the anti-Hermitian part $\Phi_{\mathrm{eff}}^{(4)}(t, \vec{r}, \vec{S})$ violates also CP invariance, i.e. invariance under Charge-Parity transformation. A violation of $\mathrm{CP}$ and $\mathrm{T}$ invariance in the spacetime with the asymmetric Kerr metric, which is analogous to a spacetime in the coordinate system rotating with an angular velocity [14], has been discussed by Hadley [34]. We would like also to mention that violation of parity and time reversal invariance in the spin-rotation interactions has been discussed by Papini [61] and Scolarici and Solombrino [62] in the model with a modified Mashhoon's potential of the spin-rotation coupling [3].

Finally we would like to discuss the results, obtained in section [V] As has been shown in [16]- 23] the wellknown non-Hermiticity of the Dirac Hamilton operator for relativistic fermions, moving in the curved spacetime with an arbitrary metric $\tilde{g}_{\mu \nu}(x)$ or in an arbitrary gravitational field, can be removed by a non-unitary transformation $\psi \rightarrow\left(\sqrt{-\tilde{g}} \tilde{e}_{0}^{\hat{0}}\right)^{1 / 2} \psi^{\prime}$, where $\tilde{g}$ is the determinant of the metric tensor $\tilde{g}_{\mu \nu}(x)$ and $\tilde{e}_{0}^{\hat{0}}$ is the vierbein field. Such a property of the Dirac Hamilton operator is also retained in the curved spacetime with an arbitrary metric, torsion and chameleon field [1, 13]. In section $\mathrm{V}$ we have shown that i) the anti-Hermitian Hamilton operator of torsionfermion interactions $\delta \mathrm{H}_{\overline{\mathrm{h}}}^{\prime}$ in the Dirac Hamilton operator $\mathrm{H}^{\prime}=\gamma^{\hat{0}} m-i \gamma^{\hat{0}} \vec{\gamma} \cdot \vec{\nabla}+\delta \mathrm{H}_{\mathrm{h}}^{\prime}+\delta \mathrm{H}_{\overline{\mathrm{h}}}^{\prime}$, obtained by means of the non-unitary transformation of the Dirac fermion wave functions $\psi \rightarrow\left(\sqrt{-\tilde{g}} \tilde{e}_{0}^{0}\right)^{1 / 2} \psi^{\prime}$, cannot be removed by any additional non-unitary transformations and ii) the existence of these anti-Hermitian torsion-fermion interactions is fully caused by the properties of the curved spacetimes with rotation, described by the metric Eq.(3).

We have also pointed out that our analysis of the Dirac Hamilton operator and a derivation of the anti-Hermitian torsion-fermion interactions are not related to the analysis of the non-Hermiticity of the Dirac Hamilton operator by means of the $\eta$-representation [24], requiring pseudo-Hermitian quantum mechanics for a description of a dynamics of Dirac fermions in curved spacetimes [24, 53, 54.

Then, we have discussed conformal invariance of the anti-Hermitian torsion-fermion interactions. As has been shown by Silenko [20], quantum field theories of massless particles, coupled to arbitrary gravitational fields or moving in curved spacetimes with arbitrary metrics, are conformal invariant under conformal transformation $\tilde{g}_{\mu \nu} \rightarrow O^{2} \check{g}_{\mu \nu}$, where $O$ is a conformal factor. The requirement of conformal invariance of quantum field theories of massive particles in curved spacetimes with arbitrary metrics can be fulfilled if and only if particle masses are changed by the conformal factor as follows $m \rightarrow O^{-1} \check{m}$ [21] (see also [25, 26]). We have shown that under the condition $m \rightarrow O^{-1} \check{m}$ the relativistic anti-Hermitian Hamilton operator $\delta \mathrm{H}_{\overline{\mathrm{h}}}$ and the anti-Hermitian effective low-energy potential $\Phi_{\mathrm{eff}}^{(4)}(t, \vec{r}, \vec{S})$ are conformal invariant.

We have discussed also an observability of the anti-Hermitian torsion-fermion interactions. As a result, we may argue that the anti-Hermitian torsion-fermion interactions can be in principle observable. First, an observability of the obtained anti-Hermitian torsion-fermion interactions is supported by their derivation, carried out on the same footing as the effective low-energy potentials $\Phi_{\mathrm{eff}}^{(1)}(t, \vec{r}, \vec{S})$ and $\Phi_{\mathrm{eff}}^{(1)}(t, \vec{r}, \vec{S})$, which have been derived earlier in [1, 13, 22, 33]. Second, an observability of the anti-Hermitian torsion-fermion interactions Eq. (22) and Eq. (38) is supported experimentally as follows. In the effective low-energy potential $\Phi_{\mathrm{eff}}^{(1)}(t, \vec{r}, \vec{S})$ the interactions

$$
\left.\delta \Phi_{\mathrm{eff}}^{(1)}(t, \vec{r}, \vec{S})\right|_{\vec{K}=-(\vec{\omega} \times \vec{r})}=-\vec{\omega} \cdot \vec{L}-\vec{\omega} \cdot \vec{S}
$$

derived also by Hehl and $\mathrm{Ni}$ [33], have been investigated experimentally by Werner, Staudenmann, and Colella 60], by Atwood et al. 2] and by Mashhoon [3]. This makes reliable in principle an observability of the anti-Hermitian torsionfermion interactions, described by the relativistic anti-Hermitian Hamilton operator Eq. (38) and the anti-Hermitian effective low-energy potential Eq.(22).

The analysis of reliability and observability of the obtained anti-Hermitian torsion-fermion interactions makes meaningful the assumption that in the rotating Universe and galaxies [50] (see also [51]) the torsion-fermion interaction $\delta \mathrm{H}_{\overline{\mathrm{h}}}$ in Eq. (38) as well as the low-energy effective potential $\Phi_{\text {eff }}^{(4)}(t, \vec{r}, \vec{S})$ in Eq. (22) might be an origin of i) violation of $C P$ and $T$ invariance in the Universe and ii) of baryon asymmetry [28].

\section{ACKNOWLEDGEMENTS}

We are grateful to Hartmut Abele for stimulating discussions. This work was supported by the Austrian "Fonds zur Förderung der Wissenschaftlichen Forschung" (FWF) under the contract I689-N16. 


\section{APPENDIX A: DETAILED CALCULATION OF THE OPERATOR $G_{\hat{j}}$}

In the Appendix we give a detailed calculation of the operator $G_{\hat{j}}$. According to Eq.(77) it is defined by

$$
\begin{aligned}
G_{\hat{j}} & =\frac{1}{2} \tilde{e}_{0}^{\hat{0}}(x)\left(\tilde{\mathcal{T}}_{\alpha \ell}^{\alpha}(x) \tilde{e}_{\hat{j}}^{\ell}(x)+\tilde{\omega}_{0 \hat{j} \hat{0}}(x) \tilde{e}_{\hat{0}}^{0}(x)+\tilde{\omega}_{\ell \hat{j} \hat{0}}(x) \tilde{e}_{\hat{0}}^{\ell}(x)+\tilde{\omega}_{\ell \hat{j} \hat{k}}(x) \tilde{e}_{\hat{\ell}}^{\ell}(x) \eta^{\hat{\ell} \hat{k}}\right) \\
& +\frac{1}{2}\left(\tilde{e}_{0}^{\hat{0}}(x)\right)^{2} \tilde{e}_{\hat{j}}^{j}(x) \frac{1}{\sqrt{-\tilde{g}(x)}} \frac{\partial}{\partial x^{j}}\left(\sqrt{-\tilde{g}(x)} \tilde{e}_{\hat{0}}^{0}(x)\right) .
\end{aligned}
$$

Using $\sqrt{-\tilde{g}}=1+U_{+}-3 U_{-}$[1] and the vierbein fields Eq.(13) we transcribe the r.h.s. of Eq. A-1) into the form

$$
G_{\hat{j}}=\frac{1}{2} \tilde{\mathcal{T}}_{\alpha \hat{j}}^{\alpha}(x)+\frac{1}{2} \tilde{\omega}_{0 \hat{j} \hat{0}}(x)+\frac{1}{2} \tilde{\omega}_{\ell \hat{j} \hat{0}}(x) K^{\ell}+\frac{1}{2} \tilde{\omega}_{\ell \hat{j} \hat{k}}(x) \eta^{\ell \hat{k}}-\frac{3}{2} \frac{\partial U_{+}}{\partial x^{\hat{j}}},
$$

where we have made the following replacements $\left(1+U_{+}+U_{-}\right) \tilde{\mathcal{T}}_{\alpha \hat{j}}^{\alpha}(x) \rightarrow \tilde{\mathcal{T}}^{\alpha}{ }_{\alpha \hat{j}}(x)$ and $\left.1+U_{+}+U_{-}\right) \tilde{\omega}_{\ell \hat{j} \hat{k}}(x) \eta^{\ell \hat{k}} \rightarrow$ $\tilde{\omega}_{\ell \hat{j} \hat{k}}(x) \eta^{\ell \hat{k}}$ and calculated

$$
\frac{1}{2}\left(\tilde{e}_{0}^{\hat{0}}(x)\right)^{2} \tilde{e}_{\hat{j}}^{j}(x) \frac{1}{\sqrt{-\tilde{g}(x)}} \frac{\partial}{\partial x^{j}}\left(\sqrt{-\tilde{g}(x)} \tilde{e}_{\hat{0}}^{0}(x)\right)=-\frac{3}{2} \frac{\partial U_{+}}{\partial x^{\hat{j}}},
$$

keeping only the linear order contributions of the gravitational, chameleon and torsion fields. Since we keep the contributions to order $O\left(K^{\hat{j}}\right)$, we get

$$
\begin{aligned}
\frac{1}{2} \tilde{\mathcal{T}}_{\alpha \hat{j}}^{\alpha}(x) & =\frac{1}{2} \mathcal{T}_{\hat{0} \hat{0} \hat{j}}+\frac{1}{2} \mathcal{T}_{\hat{\ell} \hat{k} \hat{j}} \eta^{\hat{\ell} \hat{k}} \\
\frac{1}{2} \tilde{\omega}_{0 \hat{j} \hat{0}}(x) & =\frac{1}{2} K_{\hat{j}} \frac{\partial U_{-}}{\partial t}-\frac{1}{2} \frac{\partial U_{+}}{\partial x^{\hat{j}}}+\frac{1}{2} \mathcal{K}_{\hat{j} \hat{0} \hat{0}}+\frac{1}{2} \mathcal{K}_{\hat{j} \hat{0} \hat{\ell}} K^{\hat{\ell}} \\
\frac{1}{2} \tilde{\omega}_{\ell \hat{j} \hat{0}}(x) K^{\ell} & =-\frac{1}{2} K_{\hat{j}} \frac{\partial U_{-}}{\partial t}+\frac{1}{2} \mathcal{K}_{\hat{j} \hat{\ell} \hat{0}} K^{\hat{\ell}} \\
\frac{1}{2} \tilde{\omega}_{\ell \hat{j} \hat{k}}(x) \eta^{\ell \hat{k}} & =\frac{\partial U_{-}}{\partial x^{\hat{j}}}+\frac{1}{2} \mathcal{K}_{\hat{j} \hat{\ell} \hat{k}} \eta^{\hat{\ell} \hat{k}}-\frac{1}{2} K_{\hat{j}} \mathcal{K}_{\hat{0} \hat{\ell} \hat{k}} \eta^{\hat{\ell} \hat{k}} .
\end{aligned}
$$

Plugging Eq. (A-4) into Eq. $\mathrm{A}-2$ we derive the operator $G_{\hat{j}}$ in the following form

$$
G_{\hat{j}}=-\frac{1}{2} \frac{\partial}{\partial x^{\hat{j}}}\left(U_{+}+U_{-}\right)+\frac{1}{2}\left(\mathcal{T}_{\hat{0} \hat{0} \hat{j}}+\mathcal{T}_{\hat{\ell} \hat{k} \hat{j}} \eta^{\hat{\ell} \hat{k}}+\mathcal{K}_{\hat{j} \hat{0} \hat{0}}+\mathcal{K}_{\hat{j} \hat{\ell} \hat{k}} \eta^{\hat{\ell} \hat{k}}\right)+\frac{1}{2}\left(\left(\mathcal{K}_{\hat{j} \hat{0} \hat{\ell}}+\mathcal{K}_{\hat{j} \hat{\ell} \hat{0}}\right) K^{\hat{\ell}}-K_{\hat{j}} \mathcal{K}_{\hat{0} \hat{\ell} \hat{k}} \eta^{\hat{\ell} \hat{k}}\right)
$$

Using the properties of the contorsion tensor [1] and the irreducible representation of torsion Eq. (15) we transcribe Eq.(A-5) into Eq.(26) (see also Eq.(14)).

[1] A. N. Ivanov and M. Wellenzohn, Phys. Rev. D 92, 125004 (2015); arXiv: 1511.08740 [gr-qc].

[2] D. K. Atwood, M. A. Horne, C. G. Shull, and J. Arthur, Phys. Rev. Lett. 52, 1673 (1984).

[3] B. Mashhoon, Phys. Rev. Lett. 61, 2639 (1988).

[4] H. Abele, T. Jenke, H. Leeb, and J. Schmiedmayer, Phys. Rev. D 81, 065019 (2010).

[5] T. Jenke, P. Geltenbort, H. Lemmel, H. Abele, Nature Physics 7, 468 (2011).

[6] H. Abele, T. Jenke, D. Stadler, and P. Geltenbort, Nucl. Phys. A 827, 593c (2009).

[7] T. Jenke, D. Stadler, H. Abele, and P. Geltenbort, Nucl. Instr. and Meth. in Physics Res. A 611, 318 (2009).

[8] H. Abele and H. Leeb, New J. Phys. 14, 055010 (2012).

[9] T. Jenke, G. Cronenberg, J. Bürgdorfer, L. A. Chizhova, P. Geltenbort, A. N. Ivanov, T. Lauer, T. Lins, S. Rotter, H. Saul, U. Schmidt, and H. Abele, Phys. Rev. Lett. 112, 151105 (2014).

[10] H. Lemmel, Ph. Brax, A. N. Ivanov, T. Jenke, G. Pignol, M. Pitschmann, T. Potocar, M. Wellenzohn, M. Zawisky, and H. Abele, Phys. Lett. B 743, 310 (2015).

[11] R. Lehnert, W. M. Snow, and H. Yan, Phys. Lett. B 730, 353 (2014); Erratum Phys. Lett. B 744, 415 (2015).

[12] V. A. Kostelecky, N. Russell, and J. D. Tasson, Phys. Rev. Lett. 100, 111102 (2008).

[13] A. N. Ivanov and M. Wellenzohn, Phys. Rev. D 92, 065006 (2015); arXiv: 1509.04014 [gr-qc].

[14] Yu. N. Obukhov, A. J. Silenko, and O. V. Teryaev, Phys. Rev. D 80, 064044 (2009). 
[15] Yu. N. Obukhov, A. J. Silenko, and O. V. Teryaev, Phys. Rev. D 84, 024045 (2011).

[16] Yu. N. Obukhov, A. J. Silenko, and O. V. Teryaev, Phys. Rev. D 90, 124068 (2014) and references therein.

[17] E. Fischbach, B. S. Freeman, and W.-K. Cheng, Phys. Rev. D 23, 2157 (1981).

[18] Yu. N. Obukhov, Phys. Rev. Lett. 86, 192 (2001); Fortschr. Phys. 50, 711 (2002).

[19] Yu. N. Obukhov, A. J. Silenko, and O. V. Teryaev, Phys. Rev. D 88, 084014 (2013).

[20] A. J. Silenko, Phys. Rev. D 88, 045004 (2013).

[21] A. J. Silenko, Phys. Rev. D 91, 065012 (2015).

[22] A. N. Ivanov and M. Pitschmann, Phys. Rev. D 90, 045040 (2014).

[23] A. N. Ivanov, M. Pitschmann, and M. Wellenzohn, Phys. Rev. D 92, 105034 (2015).

[24] M. V. Gorbatenko and V. P. Neznamov, Phys. Rev. D 82, 104056 (2010); Phys. Rev. D 83, 105002 (2011); arXiv: 1107.0844 [gr-qc].

[25] C. Brans and R. H. Dicke, Phys. Rev. 124, 925 (1961).

[26] R. H. Dicke, Phys. Rev. 125, 2163 (1962).

[27] V. F. Sears, in Neutron Optics, An Introduction to the Theory of Neutron Optical Phenomena and their Applications, Oxford University Press, New York, 1989.

[28] K. A. Olive et al. (Particle Data Group), Chin. Phys. A 3 38, 090001 (2014).

[29] C. Itzykson and J. Zuber, in Quantum Field Theory, McGraw-Hill, New York 1980.

[30] J. Khoury and A. Weltman, Phys. Rev. Lett. 93, 171104 (2004); Phys. Rev. D 69, 044026 (2004).

[31] D. F. Mota and D. J. Shaw, Phys. Rev. D 75, 063501 (2007); Phys. Rev. Lett. 97, 151102 (2006).

[32] L. D. Landau and E. M. Lifschitz, in LEHRBUCH DER THEORETISCHEN PHYSIK, Band II: KLASSISCHE FELDTHEORIE, Verlag Harri Deutsch, Thun und Frankfurt am Main, 2008.

[33] F. W. Hehl and W.-T. Ni, Phys. Rev. D 42, 2045 (1990) and references therein.

[34] M. J. Hadley, Europhys. Lett. 95, 21003 (2011).

[35] V. A. Kostelecky, Phys. Rev. D 69, 105009 (2004).

[36] L. D. Landau and E. M. Lifschitz, in LEHRBUCH DER THEORETISCHEN PHYSIK, Band III: QUANTENMECHANIK, Verlag Harri Deutsch, Thun und Frankfurt am Main, 2007.

[37] H. Rauch and S. A. Werner, in Neutron Interferometry, Oxford: Clarendon, 2000.

[38] H. Rauch, W. Treimer, and U. Bonse, Phys. Lett. A 47, 369 (1974).

[39] H. Rauch, A. Zeilinger, G. Badurek, A. Wilfing, W. Bauspiess, and U. Bonse, Phys. Lett. A 54, 425 (1975).

[40] G. Badurek, H. Rauch, A. Zeilinger, W. Bauspiess, and U. Bonse, Phys. Rev. D 14, 1177 (1976).

[41] G. Badurek, H. Rauch, and J. Summhammer, Phys. Rev. Lett. 51, 1015 (1983).

[42] J. Summhammer, G. Badurek, H. Rauch, U. Kischko, and A. Zeilinger, Phys. Rev. A 27, 2523 (1983).

[43] S. A. Werner, Class. Quant. Grav. 11, A207 (1994).

[44] B. Mashhoon, R. Neutze, M. Hannam, and G. E. Stedman, Phys. Lett. A 249, 161 (1998).

[45] L. H. Ryder and B. Mashhoon, Lect. Notes Phys. 562, 457 (2001); arXiv: gr-qc/0102101.

[46] Yu. Hasegawa, R. Loidl, G. Badurek, M. Baron, and H. Rauch, Nature 425, 45 (2003).

[47] S. Sponar, J. Klepp, K. Durstberger-Rennhofer, R. Loidl, S. Filipp, M. Lettner, R. A. Bertlmann, G. Badurek, H. Rauch, and Yu. Hasegawa, J. Phys. A: Math. Theor. 43, 354015 (2010).

[48] H. Rauch, Fond. Phys. 42, 760 (2012).

[49] B. Demirel, S. Sponar, and Yu. Hasegawa, New J. Phys. 17, 023065 (2015).

[50] G. Gamow, Nature 158, 549 (1946).

[51] Yu. N. Obukhov, V. A. Korotky, and F. W. Hehl, On the rotation of the Universe: arXiv: astro-ph/9705243

[52] L. L. Foldy and S. A. Wouthuysen, Phys. Rev. 78, 29 (1950).

[53] L. Parker, Phys. Rev. D 22, 1922 (1980).

[54] C. M. Bender, Rep. Prog. Phys. 70, 947 (2007).

[55] M. Arminjon and F. Reifler, Annalen der Phys. 523, 531 (2011); arXiv:0905.3686 [gr-qc].

[56] J. D. Bjorken und S. D. Drell, in RELATIVISTISCHE QUANTENMECHANIK, Bibliographisches Institut, Mannheim, Hochschultaschenbücher Verlag, 1966.

[57] A. S. Davydov, in Quantum mechanics, Pergamon Press, Oxford, 1965.

[58] Ph. Brax and G. Pignol, Phys. Rev. Lett. 107, 111301 (2011).

[59] A. N. Ivanov, R. Höllwieser, T. Jenke, M. Wellenzohn, and H. Abele, Phys. Rev. D 87, 105013 (2013).

[60] S. A. Werner, J.-L. Staudenmann, and R. Colella, Phys. Rev. Lett. 42, 1103 (1979).

[61] G. Papini, Phys. Rev. D 65, 077901 (2002).

[62] G. Scolarici and L. Solombrino, Phys. Lett. A 303, 239 (2002). 